Euskal ikerketen aldizkaria | Revue d'études basques |

Revista de estudios vascos | Basque studies review

Numéro spécial 4 | 2019

SACAZE bildumako euskal testuak (1887)

\title{
Sacaze adizkitegia
}

Gotzon Aurrekoetxea, Charles Videgain et Aitor Iglesias Chaves

\section{OpenEdition \\ Journals}

Édition électronique

URL : https://journals.openedition.org/lapurdum/2902

DOI : 10.4000/lapurdum.2902

ISSN : 1965-0655

Éditeur

IKER

Édition imprimée

Date de publication : 1 octobre 2019

Pagination : 315-337

ISBN : 9782955341339

ISSN : $1273-3830$

\section{Référence électronique}

Gotzon Aurrekoetxea, Charles Videgain eta Aitor Iglesias Chaves, «Sacaze adizkitegia», Lapurdum [Linean], Numéro spécial 4 | 2019, Sarean emana----an 12 janvier 2020, kontsultatu 22 septembre 2022. URL: http://journals.openedition.org/lapurdum/2902 ; DOI: https://doi.org/10.4000/lapurdum 2902

\section{(c) $\Theta \Theta \Theta$}

Creative Commons - Attribution-NonCommercial-NoDerivatives 4.0 International - CC BY-NC-ND 4.0 https://creativecommons.org/licenses/by-nc-nd/4.0/ 


\section{SACAZE ADIZKITEGIA}

\subsection{Sarrera}

Aditz nagusi eta laguntzailea loturik idatzirik agertzen direnetan bi zerrendetan eman dira: batetik, lexikoaren zerrendan, aditz nagusiari dagokion lekuan; eta bestetik, adizkitegi honetan, laguntzaileari dagokion lekuan. Baina bietan oso-osorik utzi da, irakurleari errazteko dagokion testuan testuinguru eta guzti irakurri gura duenerako.

Adizki bakoitzaren forma desberdinak sarrera bakarrean bildu dira. Guztira 341 sarrera eraiki dira. Sarrera hauek egitean testuan agertzen diren aldaeretan oinarrituz adizkia ortografia gaurkotuan ezarri dira. Ez dira sarrera bakarrean ezarri adizki beraren aldaera guztiak.

Adizki bakoitza ondoko ezaugarriez hornitu da:

a) Agerrera kopurua. Horrela jakin lezake irakurleak zein diren maizen agertzen diren adizkiak.

b) Bildu den testuari dagokion herri-kodea ezarri zaio segidan. Horrela, irakurleak erraz ezagutuko du non bildu den adizkia eta, halaber, inguruan dituen gainerako ezaugarriak ere ikusteko aukera izango du, hala erabakitzen badu. 


\subsection{Adizkien zerrenda alfabetikoa}

azie (15): acie (anh, etx, l-b, l-z, ost, pag), acié (zar, don), azie (a-m, izp), sinétsazié (azka), sinhetsazîe (bai), sinhetzçacie (d-g), sinhexazie (larz), sjinetjacie (iba),

azii (1): sinetsazii (bes)

azu (9): sinetsazou (gam), sinetsazu (sur), sinhetsazu (urk), sinhexaçu (beh), sinhexazu (lak), sinhexaçu (lar), açu (etxa, s-g), azou (dona)

azue (9): sinhets-assuë (luk), sinhetsaçoue (ort), sinhetsazue (bid), sinhetxaçue (b-l), sinhetxezazie (ünd), sinhexaçue (ure), açué (d-i), azue (zib, iru)

bada (2): bada (a-z, urd)

badagot (1): badagot (dom)

badaude (1): badaüde (ahe)

badenean (1): badenian (garr)

badira (2): badie (osp, urd)

baditu (1): baditu (hoz)

badizie (2): badicie (am-s, amo)

badoa (3): badoa (ald, don), badoha (ahe)

badohatzi (1): badohatci (lar)

badu (4): espadou (g-zo), espadu (a-zo), ezpadu (ida), badou (iba)

baduk (1): bauk (zib)

badute (5): badoute (ald), badute (azk, azka, mak, ure)

baita (83): baida (urr), baita (a-m, aha, aiz, arh, lek, haz, haz, hen, itu, jats, lah, dona, u-h), baita (ame, azk, azka, b-l, b-l, ban, bas, bir, lek, esp, hal, hoz, hoz, iru, iru, its, jat, jut, jut, luh, ort, d-l, sar, dono, dono, u-g), baïçaquien (eih), bayta (ur-b), beita (arü, be-l, be-l, bun, g-zo, soh, soh, ünd, esk, etxa, hau, lan, lar, l-a, l-o, mar, mau, müs, o-z, arro, d-g, irur), béita (bit, ost), beita (bun, sarr, hau, ost, pag, s-g), béita (lig, lig, ath, ath, irur), beyta (ürr, a-s, mit, z-p), bita (a-zo)

baitakot (1): beitacot (lar)

baitira (54): baitira (zar, gam, gam, jats, don, dona), béitira (ost), baitire (arh, ora, ure), baidire (bida), béitia (ath), baitira (aint, beho, esp, esp, hoz, ald, mil), baitire (b-l, u-h), béitia (hau), beitira (altz, bun, gar), beitia (l-a, men, o-z), beitira (ainh, alt, am-s, amo, ürr, bar, bar, be-l, soh, g-i, ida, larz, lar, l-o, mar, mau, mit, müs, müs, arro, s-g, dom), beytira (bil), beytire (a-s), bitira (gab), espeitié (o-z)

baitu (53): baïtou (arh), baitou (dona), baitu (azk, beho, b-l, bida, iru, izp, its, mak, urr, mil), baïtu (jats, meh, mend, u-h, ure), beitu (larz, men), beïtu (luk), bitu (u-h), espeïtu (alt, ora), espeitu (amo, lig, l-a, l-o, am-s, mar, mau, z-p, arü, oh, hau), espeytu (pag, bil), ezbaitou (eih), ezbaïtu (mug), ezpaitu (a-m), ezpeitou (gam), ezpeitu (gar, s-g), ezpeitu (g-i, mit, müs, arro, z-d, ürr, be-l, sarr, ünd), ezpitou (lekb), peitu (ort)

baitugu (1): baitugu (d-s)

baituk (1): baituk (b-l)

baituke (1): espeïtuke (altz)

baitute (3): esbeitouté (kan), ezpeitoute (a-s), baitute (ame)

baitzakian (5): baitçakian (a-m), beitçakian (hau), beitçakian (müs), beitçaquian (am-s, amo), beitzakian (1-a)

baitzakien (15): béiçakien (itu), baïtcakien (mend), baïsakien (bun), baiçakien (izt), baitçakien 
(jut, mak, ort), baitcakien (azk), bayzakien (ur-b), beitçakien (larr, ora), beitçaquien (mar), beitzakien (arh, l-o), beizakien ( $\mathrm{u}-\mathrm{h})$

baitzakin (7): baïtçakin (zar), baïtzakin (jats, dona), beïtçakin (pag), beitçakin (men), beitzakin

(gar), betçaquin (etxa)

baitzakiten (2): espeitsakiten (bun), baitsakiten (luh)

baitzauden (3): baitçauden (ure), baiçauden (its), baitzauden (d-l)

baitzazkin (1): baïtzazkin (haz)

baitzen (19): béitcènn (bit), baitcen (haz), baitcen (arh, ort, d-g, u-g), baitsen (luh), béitsen (l-s), baitzen (ame, zib), baizen (sar), beïtcen (altz), beitsën (g-z), beitcen (bard, etxa, ida, men, ora), bitzen (garr)

baitzien (14): ezpeitcien (altz), baitcakin (aint), baitcien (a-m, aha, eih), baitciren (a-m, aint, eih), baitciren (arn), baitziren (beho)

baitziin (1): beïtziin (hel)

baitzin (1): beitcin (altz)

baitziren (281): causitubaitciren (hir), baïcien (mend), baïcirénac (hen), baïciren (mug, mug, ort), baïcirenn (hen), baïtçiren (bit), béitcién (ost, ost), baïtcien (zar, beh, iba), béitcien (bard), baitcirén (ain, ain, don), baitcirènn (bit), baitciren (zar, arh, arh, lek, soh, haz, las, sen, sen, sen, dona, u-h, u-h, u-h, ure), béitsien (bun), baïtsirènn (bit), béitsirènn (bit), baïtzien (buz), baïtziren (ahe, ahe, ame, arh, buz, jats, jats, lekb, lah, lah, lah, lan, meh, bai), baicien (mend), baiciren (its), béiciren (alt), baitcien (jut, jut, leko, mend, izu, izu), baitcien- (mend), béitcien (ath, ath, ath), baitcirén (bun), baitciren (arm, azk, azk, b-l, b-l, be-l, bida, lek, get, haz, hoz, hoz, hoz, hoz, izt, izt, izt, jut, jut, jut, las, mak, mak, ort, d-g, d-i, d-i, sen, dona, dona, dona, suh, u-g, u-g, urr, uzt), baitsi'en (ban), baitsien (ban), baitsiren (ban, ald, luh), béitsiren (kan), baitzien (azka, bas, bas, bes, sur, dono), baitzi-in (aih), baitziren (ame, ame, ame, be-l, bid, d-s, zib, zib, hal, iru, izp, izp, jat, jat, lak, d-l, d-l, sar, dono, dono), baiziren (jats), beitcian (hau), beïtcién (hau, hau, ost), beïtcien (altz, altz, altz, altz, soh, hau, hau, hau, pag, pag, pag), beitcirén (ost), beïtciren (s-g), beïtsien (bun, lig, lig, lig), beïtsiren (irur), beitzien (gar), beitziren (gar, lekb), beician (müs), beicien (alt), beiciren (urd), beitçien (g-z), beitciaan (ath), beitcian (lig, mar, mau, müs), beitcién (sarr, hau), beitcien (a-zo, altz, bard, bard, be-l, g-zo, g-zo, sarr, ünd, g-i, ida, ida, laka, larr, osp, osp, men, men, men, men, men, urd), beitciren (ainh, am-s, am-s, amo, amo, anh, arü, arü, soh, ünd, g-i, ida, laka, larr, mar, mar, mau, mau, mau, mau, mau, müs, ora, ora, arro, arro, arro, arro, s-g, s-g, z-d, z-d), beitsiën (g-z, g-z), beitsien (o-z, o-z, irur, irur), beitsiren (lig, irur), beitzien (ürr, bar, gar, iho, iho, l-a, l-a, l-a, l-a, l-a, l-o, urk, urk), beitziren (alo, alo, ürr, d-s, esk, esk, ezt, hal, mit, mit, dom, dom, dom), beizien (lan), betcien (l-b), betciren (etxa, etxa), beytcien ( $z-p)$, beytciren (bil, bil, bil), beytziren (a-s, a-s, z-p), bitcien (a-zo, ilh), bitciren (gab, gab, d-g), bitzien (larz, larz), baitciren (a-m, aint, eih), batciren (aint)

baitzitien (2): baitzitien (gam), bitzitien (garr)

baitzituen (1): baicituen (lek)

baitzituzten (1): baicituzten (urr)

baitzoan (3): baitçohan (izt), baitzoan (bid), beitçoan (ort)

baitzuen (1): beitçouen (ora)

baitzuten (22): éspitsutén (itu), baitçouten (arm), baitçuten (beh, ure), béitcutien (etx), 
baïtzuten (jats), baitçuten (ort, urr), baitcuten (anh), baitzuten (aiz, iru, u-h, mil), baizuten (bir, ur-b), beitçouten (arh), beitçutien (l-z), beïtsoutén (bun), beïtsouten (bun), beitçuten (anh), bitçuten (gab), bitzuten (garr)

baizen (5): baicen (b-l, get, get, ort, d-i)

baizien (1): baïcien (aha)

bazazkien (1): baçaskienak (aha)

bazen (6): bacen (a-m, aha, aint, eih), Bacen (aha, eih)

baziren (3): Baciren (aint, beho), Baiciren (arn)

bazuzun (1): Baçuçun (a-m)

bedi (35): bédi (ain, aint, zar, g-z, itu, l-s), bedi (ahe, ainh, alo, am-s, amo, anh, arb, arn, arr, ban, lek, garr, haz, ida, iho, izp, izt, lar, l-o, luk, mug, urd, pag, arro, s-g, sen, suh, uzt), içanbedi (bit)

beza (3): beza (zib, hal, jat)

da (522): eztanic (beho), Beharda (urd, osp), Béharda (bun), behardela (urd), errandelaco (gzo), esta (eih, pag, irur, arü, ünd, lig), estenian (ainh), ezda (ame, osp, l-o), (ezda (luk), ezta (a-m, aint, mit, müs, urd, z-d, ürr, zar, sarr, g-i, hau, mar), Ezta (lar), eztenian (hal, jat), Hounada (a-s, l-b), ikaratcenda (lar), Onada (sur, ur-b), salbayada (sur, ur-b), zalbaïada (aiz), a (ünd), da (a-m, aha, aint, arn, beho, eih, aiz, aiz, aiz, aiz, ain, ain, ain, ain, ain, zar, zar, zar, ahe, ahe, ahe, ame, ame, ame, am-s, am-s, am-s, amo, amo, amo, anh, anh, anh, anh, a-z, a-z, arb, arb, arb, arb, arr, arr, arr, arr, arh, arh, arh, arh, arh, arü, arü, arü, ürr, ürr, ürr, a-s, a-s, a-s, a-s, azk, azk, azk, azk, azka, azka, azka, azka, azka, azka, altz, aih, aih, aih, b-l, b-l, b-l, b-l, ban, ban, ban, bas, bas, bas, be-l, be-l, be-l, be-l, bit, bid, bid, bid, bida, bida, bir, bir, bir, lek, lek, bun, buz, buz, buz, buz, kan, kan, soh, soh, soh, sarr, sarr, sarr, zib, zib, zib, zib, ünd, ünd, esp, esp, esp, esp, esp, gar, gar, gar, gar, gar, g-i, g-i, g-i, g-i, get, get, get, get, get, hel, hel, hel, hal, hal, hal, haz, haz, haz, hau, hau, hau, hau, hau, hen, hen, hen, hen, hen, ilh, ilh, ilh, iri, iri, iri, iru, iru, iru, iru, izt, izt, izt, izt, itu, itu, itu, itu, its, its, its, jat, jat, jat, l-b, l-b, lekb, lekb, lekb, lig, lig, lig, lig, lah, lah, lah, lah, lan, lan, lan, lan, lan, lan, lan, lan, larz, larz, larz, lar, lar, lar, lar, l-s, l-s, l-s, las, las, las, ald, ald, ald, ald, ald, ald, osp, osp, 1-o, l-o, l-o, l-o, l-o, l-o, l-o, luh, luh, luh, luh, luh, luk, luk, luk, luk, luk, mak, mak, mak, mak, mar, mar, mar, mar, mar, mar, meh, meh, meh, meh, leko, leko, leko, mit, mit, mit, mit, mug, mug, mug, müs, müs, müs, ora, ora, ora, urd, urd, urd, urd, o-z, o-z, o-z, ort, ort, ort, ort, izu, izu, izu, izu, pag, pag, pag, d-l, d-l, d-g, d-g, d-g, d-i, d-i, d-i, d-i, sen, sen, don, don, don, don, don, hir, hir, hir, hir, hir, sar, sar, sar, z-d, z-d, sur, dono, dono, bai, bai, bai, dona, dona, dom, dom, dom, suh, suh, suh, suh, ath, irur, irur, u-g, u-g, u-g, u-h, u-h, u-h, u-h, urk, ure, ure, ure, ure, ur-b, urr, urr, urr, urr, urr, uzt, uzt, uzt, uzt, mil, mil, mil, z-p, z-p, z-p), déla (bit, l-s), délaic (d-g), dën (g-z, g-z, g-z), dén (don), dénian (ur-b), danic (gab, ald, d-l), danik (beh), dela (aih, ban, ban, garr, ida, luh, luh, d-l, z-d), delaco (a-zo, bes), delako (garr, d-l), delaric (u-g), delarik (luh, sar), den (ahe, alt, arb, arr, arm, arm, altz, altz, altz, altz, aih, aih, bar, bar, bard, bas, bid, lek, lek, bes, zib, d-b, d-b, etx, gam, g-i, hel, haz, haz, iba, ida, iri, lan, lan, lan, lan, larz, larr, lar, osp, l-z, l-a, meh, meh, men, ora, urd, o-z, o-z, s-g, d-i, sen, sen, sar, sar, bai, dom, urk, uzt, mil), dena* (gab), denian (lek, ezt, z-d), denin (urk), yartzenda (azka) dabil (1): dabila (ahe)

dadien (3): egindadiela (hoz), daiela (bir), daiyela (ur-b) 
dadin (114): daïla (aha, a-z, beh, bard, iba, leko, mend, ost, d-g), dailala (a-zo, arm, g-zo, gab, hel, l-b, bai), daila (esp, izu, u-g), dailala (ezt, ilh), dailela (jut), eindaïla (urk), indaïla (gam), izandaila (azka), daïn (aiz, bas), dainn (beh), dayn (d-s), estadin (lar), ezdadin (osp), eztadin ( $z-d$, bar), dadein (mil), dadiala (etx, l-z, d-i), dadiéla (kan, hen, don), dadiela (arh, azk, beho, b-l, bida, d-s, eih, iri, iru, its, lak, luh, mak, meh, o-z, ort, hir, sur, dono, dom, u-h, urr, ald), dadi-ila (aih), dadiila (bid), dadila (alt, a-m, ame, arü, bas, ürr, a-s, altz, bar, be-l, buz, soh, sarr, d-b, ünd, esk, etxa, gar, g-i, get, jats, laka, lekb, lig, lah, lan, larz, larr, las, l-a, mau, men, mit, müs, ora, sar, dona, ath, irur, ure, bil, z-p), dadila- (mar), dadin (hal, izt, dom, mil), dadinn (hau), didala (bida)

dago (66): daü (leko), daou (ilh), ezdao (hel), çaïndago (mug), zaindô (sur), dago (ain, ame, anh, arn, a-z, arb, arr, arh, arü, eih, ürr, azk, azk, azka, b-l, ban, bida, lek, buz, kan, sarr, zib, ünd, esp, ezt, get, hal, haz, hen, iri, izt, jat, lekb, lan, lar, osp, mar, meh, mit, müs, ora, urd, ort, pag, d-l, d-i, sen, hir, sar, dom, dom, suh, u-h, ure, ur-b, urr, uzt, mil), dagoena (lar), dagola (ban), dagona (ald)

dagozu (2): dagoçu (men), dagozu (iho)

daiteke (1): daitekena (bida)

dakeat (1): dake'at (ban)

dakenat (1): (dak'enat) (ban)

dako (42): daco (zar, a-z, arm, aih, b-l, ban, bid, bun, ezt, ezt, hel, iru, jut, larz, l-s, las, luk, mak, leko, ort, izu, dono, bai, dona, suh, a-m, aint, arn, beho, eih), dako (aiz, buz, ilh, jats, l-b, lekb, lekb, ald, ora, mil, aha)

dakoat (14): dacoat (a-z, gab, hoz, iho, l-s, ora, d-g, u-g), dakoat (ame, beh, bit, d-b, ilh, larz) dakoiat (1): dacoyat (bes)

dakonat (2): daconat- (gab), dakonat (lan)

dakot (1): dacot (ezt)

dakote (2): dacoté (kan), dakote (ame)

dakozu (13): dacoçou (arm), dacoçu (a-zo, g-zo, etxa, gab), dacozu (bes, iho), dakoçu (beh, bard), dakozu (d-b, garr, urk), dakuzu (garr)

dakuat (1): dakouat (l-b)

dao (1): dao (bai)

daozka (1): daozka (meh)

daozu (1): daoçu (g-zo)

datxikola (1): datchicola (ure)

daude (7): urrundaüde (lar), daüde (iru), daude (lek, iri, lan, hir, uzt)

dauzka (5): daüska (arb, arr), daüzca (bai), daouzka (ora), dauzca (suh)

dauzu (3): daouçu (a-zo, beh), dauzü (d-b)

dee (9): déè (azka), déé (don, don), dée (don), deè (azka), dee (anh, lan, lan, ald)

deezte (1): deezte (ald)

deie (1): deïe (gar)

deio (4): déio (o-z), deïo (gar), deyo (ürr, mit)

deizie (2): deicié (a-zo), deicie (g-zo)

deizu (3): deïçu (g-zo), deiçu (a-zo, beh)

den (3): den (beho)

dena (11): déna (aint), dena (aha, arn, beho, eih), denac (eih)

deo (10): déo (hau, z-p), deo (arü, be-l, soh, sarr, ünd, l-o, urd, pag) 
dere (5): déré (irur), dere (osp, l-o, l-o, suh)

dero (6): dero (am-s, amo, g-i, l-a, z-d), dero- (arh)

detzan (1): detzan (zib)

dezagun (1): dezegun (bar)

dezan (16): estacen (larr), esteçan (ora), ezteçan (müs), eztezan (alo), désan (kan), deçala (osp), deçan (b-l, its, hir), dessala (bun), dezala (d-l), dezan (ahe, lek, zib, haz, iru)

dezaten (90): deçaten (aha, anh, arm, azk, beh, bard, bida, esp, gab, hoz, izt, jut, mak, mend, mug, ort, izu, d-i, sen, dona), déçatén (ain), déçaten (iba, lah, don), déçatenn (hen), desaten (ban, ald, luk), dessatén (bun, ost), dezaten (ame, arb, arn, arr, arh, a-s, aih, bid, buz, ezt, hel, hal, iho, iri, jat, lak, larz, meh, leko, sar, sur, dono, bai, dom, suh, u-g, u-h, mil), dezatentzat (d-l), dezeten (ur-b), diçaten (d-g), esdazaten (l-o), esdeçaten (eih), ésdéçatènn (bit), esteçaten (a-zo, mar), estéçatén (itu), estezaten (lekb), ezdéçaten (aint, a-z), ezdeçaten (ure), ezdezaten (beho, bes, gam, garr, jats), ezteçaten (a-m, amo, etxa, ilh, g-zo, l-b, las, am-s), eztéçaten (zar), eztezaten (d-b, izp, urk, aiz), tezaten (bir)

dezazie (1): chinésdéçacié (itu)

dezen (27): estécen (alt, z-p), estecen (mau, o-z, pag, ath, arü, sarr, ünd, ainh, bil), estécën (g-z), estécén (hau), estesen (ürr), estesssen (lig), estezen (l-a), eztecen (men, s-g, g-i, laka), eztezen (mit, arro, soh, esk, gar), decen (be-l), tessen (irur)

diago (1): diago (z-d)

diagozu (4): diagoçu (etx, l-z, l-a), diagozu (lak)

diakoat (2): diacoat (izp), diakoat (d-s)

diakozu (8): diacoçou (iba, jut), diacoçu (mend), diacossou (ost), diacozu (hoz, izp, lak), diakozu (d-s)

diat (99): Deytcendiat (pag), Deitcendiat (esp), itendiat (sur), diat (aiz, a-m, aha, aint, arn, beho, eih, zar, ahe, ainh, alt, alo, am-s, amo, arb, a-zo, arr, arm, arü, azka, aih, bar, bard, bas, be-l, bid, bida, bir, lek, bun, buz, kan, g-zo, soh, sarr, ünd, ünd, esk, etx, etxa, gam, gar, garr, g-i, hel, hal, haz, hau, hen, iba, ida, iru, izt, itu, jat, jats, jut, lak, laka, lekb, lig, lah, las, ald, osp, l-z, l-a, l-o, luk, mak, mar, mau, meh, leko, men, mend, müs, urd, ost, o-z, izu, s-g, d-l, d-i, don, z-d, dono, bai, dona, suh, urk, ure, urr, mil, bil, z-p)

diaudezu (1): diaudezu (d-s)

die (8): ezdiena (garr), ezdienin (bes), tiénian (beh), tielaic (ban), tienean (leko), tienen (l-b), tienian (bid, ilh)

diela (1): dielaric (arn)

dien (2): dienian (a-m, aha)

diezu (4): diéçu (itu), dieçu (altz, ida), diezou (gam)

dikozu (1): dikozou (gam)

dikuzu (1): dikuçu (altz)

dinat (24): diñat (bar), diant (sarr, gar), dignat (g-i, larr, o-z), dinat (aint, arm, be-l, buz, iri, jats, lak, lekb, bai, ure, bil, zar, hal, jat, arü, g-zo, meh), inendinat (a-zo)

dio (40): errantendio (ur-b), dio (ain, arb, arr, azk, bas, bit, bida, bir, lek, zib, esp, get, hal, haz, hen, iri, izt, its, jat, lah, lar, meh, d-l, d-i, sen, hir, sar, u-h, ure, urr, uzt), dioela (lar), dioenian (d-l), dionean (iri, sen), do (zar, bit, bir, müs)

dioat (1): dioat (a-s) 
dionat (4): diognat (arro, irur), dionat (luh, suh)

diote (18): dioté (hen, luh), diote (a-s, zib, zib, get, iri, iri, lar, lar, luh, luh, luh, luh, hir, sur), diotelaric (lar), diotenean (ahe)

diozu (18): dioçou (iba), dioçu (ainh, alt, g-z, etx, hoz, ida, laka, l-z, mau, men, s-g, ath, bil), dioozu (d-s), diozu (alo, bar, esk)

dira (468): Beardiré (ur-b), Behardia (müs, pag, sur, arü, hel), Behardira (mend, azka), béhardira (bit), Behardira (l-o), Behardire (meh, a-s, bida), ebakidienian (pag), esdia (l-s), ésdiénian (l-s), estianian (ath), estiénian (ost, bun), estinin (g-z), estirenian (larr), eztianian (etx, etxa, alo, laka, l-z), eztienian (aiz, buz, d-b), eztiénian (zar), eztienïn (urd), eztîlaïc (bai), eztilaric (las), eztinin (urk, bar, esk), Nordiren (lar), dia (be-l, soh, sarr, ünd, gar, hel, hel, hau, l-s, l-s, l-s, leko, leko, o-z, o-z, o-z, izu, pag, pag, sur, dom, irur, irur, ur-b, bil), di'elaic (ban), (di'enian) (ban), diélaïk (azka), diénïn (g-z), diénian (a-z, a-z, iba, l-s, ost), diéniann (beh), die (bas, bir, bir, bir, bes, esk, gar, g-i, l-a, müs), diela (altz, iho), dielaik (azka), dien (a-z, a-zo, altz, altz, g-zo, gam, garr, müs, o-z, o-z), dienac (altz, pag), dienean (leko), dienec (g-i, l-a), diener (osp), dienian (alo, a-zo, bas, bir, d-s, g-zo, d-b, etx, etxa, garr, iho, iho, ilh, jut, jut, l-b, laka, lig, lan, larr, osp, osp, l-z, l-o, mend, izu, d-i, d-i, sur, dono, suh, irur, z-p), dienien (bard, bard), dienin (arü, bes, sarr, ünd, urd), di-i (aih, aih, aih, aih), Diila (aih), diinian (bas, bid, hel), di-inian (aih, aih), dinian (arm, arm, ezt, gab), dira (a-m, aha, aint, arn, beho, eih, zar, zar, zar, ame, ame, am-s, am-s, amo, amo, anh, a-z, a-z, arh, arh, arm, arü, arü, ürr, ürr, ürr, a-s, azka, azka, ban, be-l, be-l, bit, bid, bid, bid, bun, bun, d-s, buz, buz, kan, kan, soh, soh, sarr, sarr, ünd, ünd, esp, ezt, ezt, etxa, gar, g-i, hal, hal, hal, iba, ida, ilh, ilh, iri, iru, izt, izt, izt, izt, izt, itu, jat, jat, jat, l-b, l-b, lig, lah, lan, lan, lan, lan, lan, larz, larz, larz, larr, lar, las, las, l-a, l-a, l-o, l-o, l-o, l-o, luh, luh, luh, luh, luk, luk, mar, meh, mit, mit, mit, müs, müs, ora, urd, urd, urd, ort, ort, izu, izu, s-g, d-g, d-i, don, don, don, don, don, z-d, z-d, dono, dono, bai, bai, bai, dona, dona, dom, dom, suh, u-g, u-h, u-h, u-h, ure, ure, uzt, mil, mil, mil, z-p, z-p), diré (ain, ain, ain, kan, itu, lah, lah, mug, ur-b, ur-b), dirélaric (ain), dirénéan (kan), dirénac (kan), dirénian (lah, don), dire (ahe, ahe, ahe, ame, arb, arb, arr, arr, a-s, azk, azk, b-l, b-l, b-l, ban, bas, bas, bida, lek, lek, zib, zib, get, get, get, get, get, haz, haz, haz, haz, hen, hen, hen, iri, iri, its, its, its, lak, lekb, lekb, lekb, lar, lar, luh, luk, mak, mak, meh, mug, mug, ora, ort, d-l, d-l, d-l, d-g, d-i, sen, sen, sen, hir, hir, hir, sar, sar, sar, dono, suh, suh, u-g, urr, urr, urr, urr, uzt, uzt), direlaïc (bai), direlaic (d-g), direlaric (eih, las, ald, u-g), diren (ame, ald, mug, mug), direnac (arn, b-l, be-l, ezt, haz, hir), direnak (arb, arr), direnean (ahe, arb, arn, zib, get, izt, its, luh, mug, d-l, sar, uzt, mil), direnec (izp), direnian (ainh, ame, anh, anh, arr, azk, lek, d-s, buz, hal, haz, iri, iru, izp, jat, lak, lekb, lan, lar, luk, mak, meh, ort, sen, suh, urr), direnin (esk), thira (gar), tirenian (aiz)

dirade (2): diadé (hau), dirade (g-i)

dirozu (3): diroçu (lig, larr, arro)

ditin (1): ditin (altz)

ditizu (2): ditiçu (ainh, ainh)

ditu (182): baratséntu (itu), Bégiatséntu (itu), Béguiatsentu (l-s), Bégiatzentu (mit), Beguiatcentu (pag), Beguiratzeintu (hal, jat), Beguiratcentu (müs), Béguiratcentu (z-p), begiratcentu (d-g), béguiratcentu (z-p), Beïratzentou (aiz), Çaintcentu (esp), Ceincentu (ort), erretenitzentu (mit), etchekitcentu (pag), guibelatcentu (esp), Saintzen'tu (ban), 
Zaintzentu (sur, azka), çaintzentu (mug), ditou (ain, ain, arh, arh, a-s, a-s, bun, buz, buz, eih, hen, lah, ald, luh, ora, sen, sen, don, don, dona, dona), ditu (ahe, ame, ame, anh, arb, arr, azk, azk, aih, b-l, b-l, bida, bida, bida, lek, zib, ezt, ezt, hal, haz, iri, iru, izt, izt, its, its, jat, larz, luk, luk, mak, mak, meh, leko, d-l, d-l, d-l, hir, sar, sar, dom, dom, u-h, urr, uzt, mil, mil), dituen (its, lar), ditueneraino (sar), tou (aha, zar, zar, bit, bit, bun, kan, kan, hel, hel, lekb, lekb, lah), tu (ahe, a-m, aint, am-s, am-s, amo, amo, anh, arn, a-z, a-z, arü, arü, ürr, ürr, ban, bas, bas, beho, be-l, bid, bid, bir, bir, bir, soh, soh, sarr, sarr, ünd, ünd, gar, gar, g-i, g-i, ilh, iru, l-b, l-b, l-b, lig, lan, lan, lan, larz, las, las, l-o, l-o, mar, mar, müs, o-z, o-z, izu, d-i, d-i, z-d, z-d, dono, dono, dono, dono, bai, suh, irur, urk, ure, ure, ur-b, ur-b, ur-b), tula (garr)

ditugu (1): ditugun (bida)

ditut (1): ditout (luh)

dituzte (7): ditouste (ald, luh, luh), ditoustenac (luh), dituz tenean (hir), dituzte (iri), dituzten (lar)

dituzu (2): ditouçou (arm), diutzu (bes)

ditzagun (1): ditçagula (lar)

ditzi (3): ditzi (jats, lak, lak)

ditzu (7): eztitzu (izp), ditçou (ora), ditzu (d-s, iho, izp, jats, lak)

diuat (1): diouat (arro)

dizi (14): Behaïci (bard), dici (arm, arm, bard, iho, jut, mend, dona), dizi (gam, izp, izp, jats), tici (arm, arm)

dizie (20): beldurraicie (gab), Behaïcie (gab), Behaïzie (jats), dicié (ainh, alt, g-z), dicie (etx, etxa, hoz, ida, ida, laka, l-z, mau, men, z-p), dizié (gam), dizie (alo, izp, lak)

dizu (17): ezdizu (bes), eztiçu (altz, men, s-g), eztizu (alo), Zaintzentizu (bes), diçu (altz, ida, larr, arro, ath), dissu (lig, irur), dizu (alo, lak, laka, urk)

doa (3): dohala (zib, lar), doua (don)

doat (3): doat (arh, g-z, u-h)

doha (1): doha (sen)

dohako (1): dohacona (lar)

doo (5): doo (aha, bid, hel, larz), do-o (aih)

doozu (1): doozu (bes)

dozu (1): doçu (gab)

du (112): ezdiinian (hel), beillatcendu (d-g), egitendu (d-g), ésdou (kan), esdu (l-s), estou (lekb), estu (amo, ora, z-p, itu, am-s), estuenia (ur-b), ézdou (don), ezdou (a-s), eztu (aiz, d-g, hir, hal, ilh, jat, las), eztuenian (bir), dou (a-s, bit, get, hen, lah, ald, ald, ald, ald, luh, luh, sen, don, don, don, dona), douélaric (ain), douénian (lah, don), douéniánn (hen), douelarik (luh), douen (lah), dounak (a-s), du (ahe, ahe, anh, a-z, arr, azk, azka, bid, bida, lek, zib, esp, ezt, get, haz, hau, iho, iri, izt, its, its, l-b, lan, larz, lar, las, l-o, mak, meh, leko, urd, izu, d-g, d-g, d-i, sar, sur, bai, dom, suh, u-g, u-g, u-g, u-g, mil, z-p), duala (lan), duen (zib), duena (iru), duenac (lek), duenean (zib, zib, haz, izt, hir, uzt), duenian (arb, arr, lak), duinian (esp), dunean (get), dou (aha), $\mathrm{du}$ (a-m, arn, beho), estienian (aha), eztinian (aint)

duk (131): étorrikoc (ur-b), Beaüc (bai), beauk (d-s), Beguirauc (hal, jat), Beirauc (hir), etorricouc (bir), handiagouk (altz), helduouc (mend), jinenduc (u-g), yéinduc (a-z), yenenduk (esp), yienduc (bes), yinenduk (sur, haz), douc (a-zo, arm, bard, bit, bun, 
kan, hel, iba, jut, lekb, lah, ald, ost, d-g, dona), douk (aiz, gam, garr, ilh, l-b, luh, don), duc (zar, ahe, ainh, alt, ame, am-s, amo, arh, arü, azka, aih, ban, bas, be-l, bid, lek, g-z, g-zo, soh, esk, etx, etxa, gab, g-i, get, hal, hen, hoz, ida, iho, iru, izp, izt, itu, jat, jats, lak, laka, lig, larz, l-s, las, osp, l-z, l-a, luk, mak, mar, müs, ora, urd, o-z, izu, pag, arro, s-g, d-i, hir, dono, bai, dom, suh, u-h, ure, z-p), duk (alo, arb, arr, beh, bar, bida, d-s, buz, sarr, zib, d-b, ünd, gar, l-o, mau, leko, men, d-l, sar, z-d, urk, mil, bil), douc (aha, eih), duc (a-m, aint, arn, beho)

dun (35): Beaun (bai), beguiraun (jat), Béhaoun (zar), ginendun (pag), doun (arm, lekb), dun (aint, a-zo, be-l, buz, sarr, ünd, g-i, iho, iri, jats, lak, lan, larr, o-z, arro, bai, suh, irur, ure, bil, zar, bar, hal, jat, luk, arü, ban, g-zo), dun- (gab)

dut (9): dout (ain, sen), dut (anh, ürr, altz, its, mit, ort, ath)

dute (33): èsdouteniann (bit), estoutino (lekb), estuténian (itu), estutienian (irur), exdute (lar), eztutelarik (d-g), douté (don), doute (hen, ald, luh, luh, luh), doutenian (luh), duté (l-b), dute (aiz, anh, zib, esp, esp, ezt, ilh, iri, lan, lar, d-g, hir, u-g), dutelaïk (azka), duten (aiz, urk), dutena (izu, urk), dutenian (izu)

dutu (5): dutu (hau, hau, osp, urd, urd)

dutuzu (2): dutuçu (ainh, ainh)

duzie (3): douçié (bit), ducie (arro, bil)

duzu (158): basauzu (iho), estuçu (mau, ath, g-z, ainh, etx, l-a), etsou (bun), ezduzu (jats), eztuçu (arro), eztuzu (urk, bar, esk), eztuçu (etxa, ida, laka, larr, l-z, bil), Hounaouçou (g-zo), Hounaouçu (a-zo), Hunaouçou (iba), hunaüçu (gab), Hunauzu (iho), itzaltzentzou (gam), lotxatuçu (ath), salbayaüçu (gab), sjalbayaouçou (iba), sjaldo (iba), düzü (d-b), douçou (arm, arm, arm, bit, iba, jut, jut, jut, dona), doussou (bun, bun, ost), douzou (gam, gam, gam, gam, gam, gam, dona), duçu (ainh, ainh, ainh, ainh, ainh, alt, alt, altz, altz, altz, altz, altz, g-z, g-z, g-z, g-z, etx, etx, etx, etxa, etxa, etxa, gab, gab, ida, ida, ida, ida, iho, laka, laka, laka, larr, larr, larr, l-z, l-z, l-z, l-a, l-a, l-a, mau, mau, mau, mau, men, men, men, mend, arro, arro, arro, arro, arro, s-g, s-g, s-g, s-g, hir, ath, ath, ath, bil, bil, bil, bil), dussu (irur, irur), dusu (l-s), duzu (alo, alo, bar, bar, bar, bar, bar, bes, d-s, d-s, d-b, esk, esk, esk, esk, ezt, ezt, ezt, ezt, hoz, hoz, izp, izp, izp, jats, jats, jats, lak, lak, lak, urk), uçu (bard, ath), uzu (aiz, garr, garr, u-h, urk, urk), Onauzu (bes)

duzue (1): duçue (hir)

egon (2): ago (itu), Ago (itu)

ezak (18): éguincéçac (z-p), eguinezac (jats), egineçak (men), eguinçac (ure), eguinzak (aiz), eintsak (bard), eisak (leko), Eizak (urk), eiçak (esp), Intzac (haz), isac (l-s, ald), izac (meh), Kasouiçcac (arm), eçak (mit, bil), esak (ürr), zak (zib)

ezan (4): eguinezan (jats), eguinçan (ure), Kasouïçan (arm), eçan (bil)

ezazie (18): essassie (o-z), éçacié (g-z, hau), eçacie (arü, g-i, larr, müs, ora, z-d), ecacie (mar,

ath), esasie (mit), ezazie (alo, buz, sarr, esk, gar, lekb)

ezazien (1): éçacién (z-p)

ezazu (10): essassu (irur, lig), eçaçu (ainh, ida, l-a, men), eçacu (mau), ezazu (ürr, be-l, soh) ezazue (6): atçué (izt), Emaçue (its), cacie (izu), ezazue (ame), sasoué (kan), sasoue (luh) hadi (43): Abisadi (z-d, etxa, g-i, alt, alo, lig, larr), Abizadi (ath, hau), beguiadi (hau), ai (altz, altz, altz, g-zo, g-zo, lig, leko, o-z, ath, irur), ai (a-zo, bid, bir, bir, leko, men), ay (ur-b), hadi (ainh, am-s, amo, arü, bar, g-z, sarr, ünd, osp, l-a, mau, o-z, izu, pag, arro, s-g) 
nauzu (2): nuçu (lek), nuzu (dom)

nezazu (1): nesasou (ald)

nezazue (1): nezazue (sar)

nihoak (1): nihoak (zib)

niok (1): nioc (bir)

nion (2): niok (sur, ur-b)

tizi (12): titçu (alt, alt, alt, etxa, etxa, men, men, bil, bil), titci (bard), titsi (bard), titzu (urk)

tizu (31): Béguiatcentiçu (etx), tiçu (altz, altz, g-z, g-z, etx, ida, ida, laka, laka, larr, larr, l-z, l-z,

l-a, l-a, mau, mau, arro, arro, s-g, s-g, ath, ath), tizu (alo, alo, bar, bar, be-l, esk, esk)

titzu (1): zaintzentitzu (bes)

tutzu (12): tutçu (alt, bard, etxa, gab, men, ath, bil), tutzu (alo, bes, hoz, urk, urk)

tuzi (1): tüzi (d-b)

tuzte (3): touzté (don), tuste (lar), tuzte (ahe)

tuzu (25): tüçu (g-z), touçou (arm), tuçu (altz, g-z, etx, etx, ida, laka, laka, lig, larr, larr, l-z,

l-z, mau, mau, arro, arro, s-g, s-g), tuzu (bar, bar, esk, esk, jats)

tzi (5): Behartci (beh), Behartzi (gam), beilatzenzi (garr), beyratzentzi (d-s), Beïratcentci (iba) tzie (3): Béhartcié (ost), Behartcié (a-zo), Behartcie (g-zo)

zabilan (1): çabilan (urd)

zabiltzan (1): zabiltzan (d-1)

zadien (1): çadien (dono)

zaezte (1): zaezte (bir)

zagoen (10): çagoen (b-l, lar, lar), çagouelaric (l-a), çagouen (l-a), sagouen (kan), zagoelaric (hoz), zagoen (d-l, d-l), zaguela (d-l)

zagon (26): çagola (ure, urr), çagolaric (b-l), çagolarik (bida), çagon (ain, ora, d-g, d-i, u-g, ure), cagoela (lar), sagola (luh), sagularik (l-o), zagola (hal, jat, uzt), zagolaric (lekb), zagon (ahe, arh, lek, d-b, haz, iri, lan, u-h, uzt)

zagotzen (1): zaotzen (ahe)

zagotzun (1): zagotzun (bar)

zagozin (1): çagocin (azk)

zaien (2): zaien (hal, jat)

zaion (2): zaiona (hal, jat)

zaioten (7): çaioten (ort, ort), tzakoten (dono), tzayoten (dono), zaïoten (ahe), zayoten (a-s, beho)

zaitezte (1): zaitezte (ame)

zaitzen (1): Zaitzen (meh)

zaizte (9): çaïste (hen), çaizte (bida, sen, urr), zaïste (ahe), zaïzte (bas, haz), zaiste (zib), zaizte (d-1)

zaizten (1): zaizten (urk)

zak (3): çac (sen), tçac (gab, jut)

zakian (9): çakian (g-i, laka, lig, osp, s-g), çakianac (z-d), çaquian (alt, soh), caquian (be-l)

zakien (90): baçaquien (hir), bazakien (sar), bazakienak (sur), etsaquien (o-z), etsakien (irur, lig), etzakien (mend, mit), etçakien (urd, pag, s-g, z-d), etzakien (arü, ürr), etçaquien (be-l), etçakien (soh), etzakien (sarr, gar), etçakien (g-i), étçakién (hau), etzaquien (la), çakién (ain), çakiéna (a-z), çakiénak (beh), çakiènn (bit), çakien (ainh, a-zo, arm, b-l, bard, g-zo, gab, iba, ilh, lar, las, osp, urd, d-g, don, u-g, ure), çakienac (its, ald, 
izu), çaquien (iru, d-i), çaquiena (urr), cakien (anh, l-b), sakién (ost), sakien (kan, lekb, luh, z-p), sakienic (irur), saquiéna (l-s), saquien (o-z), saquienn (hen), zakien (ahe, arb, arr, azka, ban, beho, buz, d-b, ünd, ezt, gam, get, hal, hoz, iho, iri, izp, jat, larz, meh, leko, d-l, dono, dom, mil), zakienac (ame, arn, lak, luk, suh), zakienak (a-s) zakiin (4): zakiin (bas, bes), zaki-in (aih), zakiinac (hel)

zakin (19): çakin (arü, altz, esp, mau, bil), çakinac (lah), çakinak (arro), sakin (ürr, mit), sakinac (ath), saquïn (g-z), zakin (alo, bar, bid, sarr, ünd, esk, urk), zaquîn (bai)

zakiten (88): bazakiten (iri), çaquiten (eih), etçaquiten (aha), etçakiten (a-m), eztçakiten (aint), étsakiten (kan, itu), etxaquiten (arr), etzakitela (hoz), etçakiten (müs), etzakiten (aiz), etçakiten (izu, a-z), etzakiten (sur, dono), etzaquiten (bai), etzakiten (dom), etzaquiten (arb), etzakiten (u-g, urk), etçakiten (ure), etzakiten (z-p, a-s), étçakitén (ain), etçakiten (zar), etzakiten (bid), etzaquiten (bir), etzakiten (buz, d-b, hel), etzaquiten (hal), etçakiten (ilh), etzakiten (iru), etcakiten (izt), etzaquiten (jat), etçakiten (l-b), etzakiten (lekb), etçakiten (lan), etzakiten (larz, lar), etçakiten (las), etzakiten (l-o, luk), exakiten (d-g), ezakiten (ur-b), ezçakiten (b-l), çakiten (anh, azk, bida, lek, esp, haz, its, mak, leko, mug, ora, ort, sen, don, dona), çaquiténn (hen), çaquiten (am-s, amo, d-i, hir, urr), sakiten (lah, ald), tsakiten (ban), tzakiten (uzt), zakiten (ahe, ame, arh, arn, azka, aih, bas, beho, zib, ezt, iri, meh, sar, suh, u-h, mil), zaquiten (get)

zakizien (29): eshakicien (bard), etsaquiciën (g-z), etçaquicien (alt, mau), etçakicien (men, arro), etçakizien (ath), etzaquicien (a-zo), etçakicien (altz), etzakizien (bar), étçakicièn (bit), etzakizien (bes), etzaquicien (g-zo), etzakizien (esk), etçakicien (ainh), etçakizien (etx), etzakizien (gam, garr), etçakicien (ida), etzakicien (iho), etzakizien (alo), etçakizien (laka), etçakicien (larr, l-z, mar, bil), eztçaquicien (etxa), çakicien (beh), tçakicien (gab)

zakizin (1): ezçakizin (arm)

zakizkan (2): çakizkan (sen), zakizcan (uzt)

zakizkin (1): zaquisquinac (bir)

zakon (5): çacolaric (b-l), çacon (b-l), zacola (izp), zakolaik (urk), zakolaric (ame)

zakonat (1): saconat (iho)

zakoten (19): çacoten (b-l, b-l, lek, leko), çakoten (mak, mak, mug, mug, ora, ora), cacoten (leko), cakoten (ora), sacotén (itu), sacoten (kan, kan), zacoten (aih, dom, dom), zakoten (mil)

zakotzien (1): zacotzien (bes)

zakozien (6): etzakozien (d-b), çacocien (etxa), çakocien (bard, bard, etxa), zacozien (bes)

zan (1): tçan (gab)

zarete (11): çarété (ain), çarete (azk, b-l, mak, ort, hir), zaëte (meh), zarete (arb, arr, sar, uzt)

zarezte (2): çaresté (lah), zareste (get)

zarraizkoten (2): zarraizcotela (hal, jat)

zatortzin (1): zathortzinak (d-1)

zauden (17): çaüdelaric (mug), çaouden (ora, sen), çauden (eih, its, lar, las, hir), zauden (azka, b-l, zib, hal, iri, jat, dono, dom, suh)

zaudetzun (2): çaoudetçun (etx, l-z)

zaukan (1): zaukan (zib)

zauten (1): ézçauténn (hen)

zazie (18): sinhetsacie (leko), sinetsacie (arm), sinhetsacié (ilh), sinhezcasié (l-o), çacie (bard, 
gab, jut, las), zazié (u-h), zazie (aiz, a-s, d-s, jats, lan, meh), çacie (aha), zacie (arn), zazie (beho)

zazii (2): zacii (hel), zazii (bas)

zazkoten (2): çascoten (eih), zazcoten (aih)

zazte (1): sasté (ur-b)

zazu (13): sinhetsassou (bun), çaçou (ain), çaçu (am-s, amo, azk, esp, its, mug, urr), zazu (aih, suh, u-g, mil)

zazue (16): chinetch-çaçoue (sen), çaçouë (hen), çaçoue (lah), çaçué (a-z), çaçue (mak), zazue (arb, arr, bir, get, hal, haz, iri, jat, d-l, dono), sasuez (ur-b)

ze (13): çè (bit), ce (bard, bid, ilh, izt, l-b, leko, mend), zé (gam), zê (sur), ze (bes, d-b, larz) zedin (1): etsein (urd)

zeen (41): errantzeen (larz), céen (zar, alt, a-zo, ilh, ilh, o-z, dona, ath), ceen (am-s, amo, anh, altz, altz, hel, iru, lar, leko, izu, dona, ure), etceren (g-i), se'en (ban), Seen (a-zo), tzeen (hel), zeên (sur), ze'en (ban), zeen (arh, arh, bid, bes, d-s, buz, bai, u-h, u-h), céén (aint), céen (aint), ceen (aha, arn), zeen (beho)

zeetan (2): ceetan (arn), ceeten (aha, arn)

zeeten (14): çéétenn (bit), céétènn (bit), cééten (zar), céeten (dona), ceeten (anh, iru, izu, ure), se'eten (ban), tzeeten (hel), zeeten (bid, suh), cééten (aint), ceeten (aha, arn)

zeezoun (1): çééçoun (bit)

zeezten (1): zeezten (buz)

zeien (17): ceien (urd, urd), ceyen (am-s, amo, be-l, soh, mit, pag, pag, s-g, s-g, ure, z-p, z-p), seien (urd), seyen (ürr, mit)

zeiezun (2): ceieçun (mar), zeiezun (urk)

zeiien (1): zeiïen (gar)

zeiten (3): céiten (a-z), ceiten (ilh, l-b)

zeitezten (1): ceitesten (ame)

zeizien (3): ceïcien (beh), zeizien (d-b, garr)

zeizun (1): ceïçun (gab)

zekiten (2): etchekiten (bar, ida)

zen (1440): baçén (itu), baçènn (bit), bacén (ain, hau, hen, don, don, don), Bacén (hau),

Bacénn (hen), bâcebat (alt), bacen (zar, am-s, amo, anh, a-z, arh, arü, b-l, be-l, bida, bir, lek, soh, sarr, ünd, esp, g-i, get, ilh, its, l-b, lah, l-a, mak, müs, ora, urd, o-z, ort, izu, pag, s-g, d-g, d-i, sen, z-d, dono, dona, u-g, ure, ur-b, ur-b, uzt, z-p, z-p), Bacen (am-s, amo, be-l, soh, sarr, ünd, esp, g-i, get, ilh, lig, lah, mau, müs, ora, ort, izu, pag, s-g, d-i, sen, dono), basen (ürr, ban, l-o, luh, luk, mit), Basen (hel, ald), bassén (bun, itu), Bassèn (bit, bun), bassen (lig, l-s, irur), Bassen (o-z, irur), bazen (aiz, azka, bid, buz, ezt, gar, hel, iru, lekb, sar, bai, dom, suh, urk), Bazen (arh, ürr, a-s, azka, ezt, gar, lekb, larz, luk, mit, suh, u-h, urk, mil), aïcén (hau), aïcen (pag), alhacélaric (itu), alhacen (pag, g-i, mar), alhancen (ilh, l-b), alhatsen (bun), alatcen (meh), alhatcen (men, ost), alatcen (sen), alhatcen (z-d, dona), alhatzen (mil), alhatcen (bil, azk), alhatzen (azka), alhatcen (bard), alhatzen (bas, bes, ezt), alhatcen (gab), alhatzen (hel), alhatcen (hoz), alhatzen (iru), alhatcen (jut, las, mak), alhatcencen (leko), alatzenzen (sur), alhazen (dom, a-s), aricen (d-g), arizen (aiz, a-s), batzen (aih, larz), beracen (mug), emancen (get), emansioten (ur-b), emantxen (dono), emantzen (haz), emantcen (hir), emantzen (dom, urk, arb), emanzen (ur-b), emantcen (esp), emantzen (larz), 
érantsen (l-s), espekatzenzela (sur), estaliacen (leko, mug), etsén (itu), etzen (l-a), etcen (uzt), gancen (ort), gançen (ur-b), ganzen (get), haïcen (mug), haicen (hir, esp), haizen (urk), haricen (dono), houracen (ort), hourazen (aiz), -hourazen (a-s), -huazen (larz), huracen (get), -huranzen (mil), hurazen (azka), iarricen (d-g), igaitentzen (aiz), igarançenn (bit), ikharatoussén (bun), ikaratsen (luh), ikharatucen (leko, mug), ikharatuzen (aiz), ikaratuzen (sur), ikharatcen (urd), ikharatcén (don), ikharatcen (hir, uzt), ikharatzen (a-s, azka), ikaratcen (esp), ikharatzen (gam), ikaratcen (get), ikaratcénn (hen), ikharatcen (iri, lan), ikharatzen (l-o), ikaratzen (luk), ikharatcen (mar), isantsén (l-s), Içancen (pag), Izancen (get), içancen (itu), jarricen (ünd, ilh), juàtcénn (hen), lothucen (pag), lozelaïk (garr), lurracen (ort), ohartouzen (hel), phasatouzen (a-s), phasatuzen (bes), pasatuzen (sur, ur-b), phasatuzen (azka), passatoussén (bun), passatouzen (gam), passatucen (mend), pazatucen (ur-b), phazatzentzen (garr), Tantugucen (ort), uiancen (d-g), choutitouzen (a-s), youantzen (a-s), youanzen (a-s), youantcen (l-b), yuantzen (sur), yuanzen (urk), yuantcen (uzt), yuanzen (azka), yuantcen (esp), yuantzen (lan, larz), çéla (bit), çènn (bit, bit), (çen) (luk), çen (luk, luk, luk, luk, luk), cèla (bun), céla (ain, zar, a-z, hau, hen, iba, lah, lah, ost, ath), cèn (ain), cën (g-z), cén (ain, ain, ain, ain, ain, ain, ain, ain, hau, hau, hau, hau, hau, hau, hau, hau, hau, hau, hau, ost, don, don, don), cènn (bit, bit, bit), cénn (hen, hen, hen, hen, hen, hen), cènne (bit), cela (ainh, alt, am-s, amo, anh, arb, a-zo, arr, arh, arm, arm, arü, azk, altz, altz, beh, beh, b-l, b-l, bard, bard, be-l, bida, lek, g-z, g-zo, soh, esp, etx, etxa, gab, g-i, get, hen, hoz, iba, ilh, iri, iru, izt, itu, its, jut, l-b, laka, larr, lar, las, osp, l-z, l-a, mak, mar, mau, meh, leko, men, mend, mug, mug, müs, ora, ort, izu, pag, arro, s-g, d-g, d-i, sen, hir, z-d, dono, dono, dona, suh, u-g, ure, urr, uzt, bil, bil), celaik (altz), celaric (meh, meh, sen), cen (ain, zar, zar, zar, zar, zar, zar, zar, zar, zar, zar, zar, ainh, alt, alt, am-s, am-s, am-s, am-s, am-s, am-s, am-s, am-s, am-s, am-s, am-s, amo, amo, amo, amo, amo, amo, amo, amo, amo, amo, amo, anh, anh, anh, anh, anh, anh, anh, anh, anh, a-z, a-z, a-z, a-z, a-z, a-z, a-z, a-z, a-z, arb, arb, arb, arb, arb, arb, arb, a-zo, arr, arr, arr, arr, arr, arr, arh, arh, arh, arh, arh, arm, arü, arü, arü, arü, arü, arü, arü, arü, arü, arü, ürr, azk, azk, azk, azk, azk, azk, azk, azk, azk, azk, azk, azk, altz, beh, b-l, b-l, b-l, b-l, b-l, b-l, b-l, b-l, b-l, ban, ban, bard, bard, be-l, bel, be-1, be-l, be-l, be-1, be-l, be-1, be-1, be-1, be-1, be-1, bid, bid, bid, bid, bida, bida, bida, bida, bida, bida, bida, bida, bida, bir, bir, bir, bir, bir, bir, bir, bir, lek, lek, lek, lek, lek, lek, lek, lek, lek, g-zo, soh, soh, soh, soh, soh, soh, soh, soh, soh, soh, soh, soh, sarr, sarr, sarr, sarr, sarr, sarr, sarr, sarr, sarr, sarr, ünd, ünd, ünd, ünd, ünd, ünd, ünd, ünd, esp, esp, esp, esp, esp, etx, etxa, gab, g-i, g-i, g-i, g-i, g-i, g-i, g-i, g-i, g-i, get, get, hal, haz, haz, haz, haz, haz, haz, haz, haz, hau, hoz, ida, ilh, ilh, ilh, ilh, ilh, ilh, ilh, iri, iri, iri, iri, iri, iri, iru, iru, iru, iru, iru, iru, iru, iru, iru, iru, iru, izt, izt, izt, izt, izt, izt, izt, izt, izt, izt, izt, izt, itu, its, its, its, its, its, its, its, its, its, its, its, its, jat, jat, jut, l-b, l-b, l-b, l-b, l-b, l-b, laka, lah, lah, lah, lah, lah, lah, lah, lah, lah, lan, lan, larr, lar, lar, lar, lar, las, las, las, las, las, las, las, las, las, las, osp, osp, osp, osp, osp, osp, osp, osp, l-z, l-a, l-a, l-a, l-a, l-a, l-a, l-a, l-a, luk, luk, luk, luk, mak, mak, mak, mak, mak, mak, mak, mak, mak, mak, mar, mar, mar, mar, mar, mar, mar, mau, meh, meh, meh, meh, meh, meh, meh, meh, meh, meh, leko, leko, leko, leko, leko, leko, men, mit, mit, mug, mug, mug, mug, mug, mug, mug, müs, müs, müs, müs, müs, müs, müs, müs, ora, ora, ora, ora, ora, ora, ora, ora, ora, ora, urd, urd, urd, o-z, o-z, o-z, o-z, o-z, o-z, ort, ort, 
ort, ort, ort, ort, ort, ort, izu, izu, izu, izu, izu, izu, izu, izu, izu, izu, pag, pag, pag, pag, pag, pag, arro, s-g, s-g, s-g, s-g, s-g, s-g, s-g, d-g, d-g, d-g, d-g, d-g, d-g, d-i, d-i, d-i, d-i, d-i, d-i, d-i, sen, sen, sen, sen, sen, sen, sen, sen, sen, sen, sen, don, don, hir, hir, hir, z-d, z-d, z-d, z-d, z-d, z-d, z-d, z-d, z-d, z-d, dono, dono, dono, dono, dono, dono, dono, dono, dono, dono, bai, dona, dona, dona, dona, dona, dona, dona, dona, dona, dona, dona, suh, suh, suh, suh, suh, suh, suh, suh, suh, ath, ath, u-g, u-g, u-g, u-g, $\mathrm{u}-\mathrm{g}, \mathrm{u}-\mathrm{g}, \mathrm{u}-\mathrm{g}, \mathrm{u}-\mathrm{g}, \mathrm{u}-\mathrm{g}$, ure, ure, ure, ure, ure, ure, ure, ure, ure, ur-b, ur-b, ur-b, ur-b, urr, urr, urr, urr, urr, urr, urr, urr, urr, urr, urr, uzt, uzt, uzt, uzt, uzt, uzt, bil, z-p, z-p, z-p, z-p, z-p, z-p, z-p), cena (lek, get, its), cenac (mug), cenean (arn, bida, mug), cenian (urr), cenn (hen), izantzen (aiz, azka, larz, mit), Izantzen (mil), Izantzenê (sur), jouansén (itu), jouantsen (o-z), jouantzen (mit), sèla (bun), séla (kan, l-s, irur), sén (bun, bun, bun, bun, bun, bun, bun, itu, itu, itu, itu, l-s, l-s, l-s, l-s, l-s, l-s, l-s, l-s), Sën (g-z, g-z), Sén (hau), sela (lig, ald, l-o, mit, urd, urd, o-z, z-p), sen (ürr, ürr, ürr, ürr, ürr, altz, altz, ban, ban, ban, ban, ban, ban, ban, ban, bun, bun, kan, kan, kan, kan, kan, kan, kan, kan, kan, kan, l-b, lig, lig, lig, lig, lig, lig, lah, l-s, ald, ald, ald, ald, ald, ald, ald, ald, l-o, l-o, l-o, l-o, l-o, l-o, l-o, luh, luh, luh, luh, luh, luk, mit, mit, mit, mit, urd, urd, urd, urd, o-z, o-z, o-z, o-z, o-z, o-z, irur, irur, irur, irur, irur, irur, irur, irur, irur, z-p), Sen (ame, altz, g-zo, arro, ath), tcen (bida, bida, uzt), tsen (arr), txen (arr), tzen (arb, ürr, gar), yarrizen (aiz, sur), zén (bun), zela (aiz, ahe, alo, ame, arh, ürr, a-s, azka, aih, bar, bas, bid, bir, bes, d-s, buz, sarr, zib, d-b, ünd, esk, ezt, gam, gar, garr, hel, hal, haz, haz, iho, izp, jat, lak, lekb, lan, larz, luk, d-l, sar, sur, bai, dom, u-h, u-h, urk, ur-b, mil, mil), zen (ahe, ahe, ahe, ahe, ahe, ahe, ahe, alo, ame, ame, ame, ame, ame, ame, ame, ame, arb, arr, arh, arh, arh, ürr, ürr, ürr, a-s, a-s, azka, aih, aih, aih, aih, aih, aih, aih, aih, bar, bas, bas, bas, bas, bas, bas, bas, bas, bas, bas, bid, bid, bid, bid, bid, bid, bid, bir, bir, d-s, buz, buz, buz, buz, buz, buz, buz, buz, buz, buz, buz, zib, zib, zib, zib, zib, zib, zib, zib, zib, d-b, ünd, esk, ezt, ezt, ezt, ezt, ezt, ezt, ezt, ezt, ezt, ezt, gar, gar, gar, gar, gar, gar, gar, gar, gar, gar, garr, garr, get, get, hel, hel, hel, hel, hel, hel, hel, hel, hel, hal, hal, hal, hal, hal, hal, hal, hal, hal, hal, haz, iho, iri, iri, izp, jat, jat, jat, jat, jat, jat, jat, jat, jat, jats, lak, lekb, lekb, lekb, lekb, lekb, lekb, lekb, lekb, lekb, lekb, lig, lig, lah, lah, lan, lan, lan, lan, lan, lan, larz, larz, larz, larz, larz, larz, las, osp, luk, mit, mit, müs, d-l, d-l, d-l, d-l, d-l, d-l, d-l, d-l, d-l, d-l, sar, sar, sar, sar, sar, sar, sar, sar, sar, sar, sur, sur, sur, bai, bai, bai, bai, bai, bai, bai, bai, bai, bai, dom, dom, dom, dom, dom, dom, dom, dom, u-h, u-h, u-h, u-h, u-h, u-h, u-h, u-h, u-h, u-h, urk, urk, urk, urk, mil, mil, mil, mil, mil, mil, mil), zenian (garr), sen (aint), cen (a-m, aha, aint, arn, beho, eih), zen (beho), cen (arn), céla (aint), cela (a-m, aha, arn, eih), zela (beho)

zeren (2): ceren (eih), zeren (beho)

zereten (1): cereten (las)

zerezten (1): zerezten (ezt)

zertzeen (1): zertzien (iho)

zeten (3): ceten (izt, izt), zêten (bai)

zezaten (1): cezaten (get)

zezin (2): cecin (arm, arm)

zezteen (1): zezteen (larz)

zezten (1): zeztelaric (ezt)

zi (35): Beguiatcentci (izu), estzi (d-s), etci (d-g, mend), étci (ost), etci (a-zo, bard, g-zo), etzi 
(d-b), Etzi (garr), etci (hoz), étci (iba), Saintzentzi (hoz), Zaintzentzi (iho), ci (mend), tci (a-zo, a-zo, a-zo, beh, g-zo, g-zo, g-zo, gab, jut, jut, mend, ost, ost, ost), tzi (d-b, d-b, gam, garr, jats), zi (aih)

ziade (6): ciadé ( $z-p)$, ciade (etx, l-z, l-a), siadé (irur), siade (lig)

ziadeie (1): ciadeyé (s-g)

ziagotzun (1): ciagotçun (ida)

ziagozun (2): ziagozun (izp, lak)

ziakizien (8): étciakicién (ost), etciakicien (hoz), etziakizien (izp, jats), ezciakicien (iba), ciakicien (jut), ziakizien (d-s, lak)

zian (37): arician (ure), cialaric (pag), cian (am-s, am-s, amo, amo, be-l, be-l, soh, soh, g-i, g-i, g-i, hau, hau, osp, osp, osp, l-a, l-a, müs, müs, müs, müs, müs, s-g, z-d, z-d, z-d, irur), ciann (hau), sian (lig, o-z, irur, irur), zian (gar, l-a)

ziaudezun (2): ciaudezun (hoz), ziaudezun (iho)

ziazte (2): ciasté (etxa), siazte (l-o)

zide (12): cidé (altz, hau, ath), cide (laka, larr, mau, men, müs), sidé (alt, o-z), zide (alo, gar) zidie (11): cidié (g-z), cidie (arü, sarr, ünd, urd, pag, bil), zidie (ürr, bar, esk, mit)

zie (4): tcié (a-zo), tcie (a-zo, g-zo, jut)

zie/ozun (1): zie/ozun (bar)

ziela (1): ciela (arn)

zielarik (1): cielaik (a-m)

zien (690): Cien (arn, eih), arizien (azka), aricien (l-b), arizirelaik (azka), ari_cirelaric (s-g, iri, las), aricirelaric (mar), ariziren (aiz), etsien (o-z, irur, lig), Etsien (urd, o-z, irur, lig), etcien (arü), etzien (mit), etcien (urd, pag, s-g, z-d, ath), etzien (ürr), etcien (be-l, sarr), etzien (gar), etcien (g-i, hau), etzien (l-a), Etzien (mit), Etcien (pag, s-g, z-d, arü), Etzien (ürr), Etcien (be-l, soh, sarr), Etzien (gar), Etcien (g-i), Etcién (hau), Etzien (1a), ikhoussissien (bun), itzitzien (garr), çien (luk, luk, ur-b), ciélaïc (zar, iba), ciélacotz (a-z, iba), ciën (g-z), cién (a-z, a-z, g-z, hau, hau, hau, hau, hau, hau, iba, ost, ost, don, don), ciéna (ainh, alt, hau, o-z), ciénac (alt, alt, etx, iba, iba, iba, lah), ciénak (beh, beh, beh, ost), ciénétaïc (alt), ciénian (zar, alt), ciela (anh, ilh, l-b), cielaïc (pag, irur), cielaïk (altz, leko), cielaco (l-a), cielaic (ainh, bid, sarr, ida, mau, müs), cielaik (laka), cielako (men), cielaric (am-s, am-s, am-s, amo, amo, amo, ünd, g-i, ida, larr, l-a, ora, suh), cielarik (arü), cien (a-m, aha, aint, arn, beho, zar, zar, zar, zar, ainh, ainh, alt, alt, am-s, amo, anh, anh, anh, anh, anh, anh, anh, anh, anh, a-z, a-z, a-z, a-z, a-z, a-z, a-zo, a-zo, arh, arü, arü, arü, arü, arü, arü, arü, altz, altz, beh, beh, beh, bard, bard, bard, bard, be-l, be-l, be-l, be-l, be-l, be-l, be-l, bid, bid, bid, bid, bid, bir, bir, bir, bir, bir, bir, g-z, g-zo, g-zo, g-zo, soh, soh, soh, soh, soh, soh, soh, sarr, sarr, sarr, sarr, sarr, sarr, sarr, ünd, ünd, ünd, ünd, ünd, ünd, ünd, etx, etx, etx, etxa, etxa, g-i, g-i, g-i, g-i, g-i, g-i, g-i, g-i, g-i, g-i, hau, hau, hau, hau, hoz, ida, ida, ida, ilh, ilh, ilh, ilh, ilh, ilh, ilh, ilh, izt, jut, jut, jut, l-b, l-b, l-b, l-b, l-b, l-b, l-b, l-b, laka, larz, larr, larr, las, osp, osp, osp, osp, osp, osp, osp, osp, osp, osp, l-z, l-z, l-z, l-a, l-a, l-a, l-a, l-a, l-a, l-a, l-a, mar, mar, mar, mar, mau, mau, leko, leko, leko, leko, leko, leko, leko, men, men, mend, mend, mend, mit, mug, müs, müs, müs, müs, ora, urd, urd, urd, o-z, izu, izu, izu, izu, izu, $i z u, i z u, i z u, i z u, i z u, i z u, p a g$, pag, pag, pag, pag, pag, pag, pag, pag, arro, s-g, s-g, d-g, d-g, d-g, d-i, d-i, d-i, d-i, z-d, z-d, z-d, z-d, dona, dona, suh, suh, suh, ath, ath, ath, ath, irur, irur, irur, irur, u-h, u-h, ure, ur-b, ur-b, ur-b, z-p, z-p, z-p, z-p, z-p, z-p, 
z-p, z-p, z-p, z-p, z-p, z-p), cien- (pag), Cien (gab), ciena (arü, sarr, ünd, etx, g-i, l-z, men, arro, s-g), cienac (aha, a-m, am-s, amo, a-zo, arü, azka, beho, be-l, be-l, be-l, bir, bir, bir, g-z, g-zo, soh, soh, soh, sarr, ünd, etx, gab, gab, gab, g-i, g-i, g-i, ida, ida, jut, lig, larr, l-z, l-z, l-a, mend, müs, izu, izu, izu, pag, pag, ath, ur-b, ur-b, ur-b), cienak (aiz, bard, ilh, ilh, ilh, l-b, l-b, l-b, laka, laka, mau, bil), cienean (leko), cienek (leko), cienen (aha, mau), cienez (ath), cienian (a-zo, bid, bir, bir, g-zo, etx, etxa, g-i, ilh, l-b, l-z, müs, d-i, dono), cienien (bard), cienin (ainh, arü, g-z, sarr, ünd, laka, pag, bil), cienn (ilh), izantzien (sur), si'elay'ic (ban), si'en (ban, ban, ban), si'en) (ban), si'enac (ban), siélaïc (l-s, o-z), siélacos (l-s), sièn (bit, bun), sién (bun, bun, itu, itu, l-s, l-s, l-s, l-s, l-s, l-s, l-s, l-s, l-s), siénac (bun, l-s), siénétic (l-s), siènian (ur-b), siénian (itu), sielarik (lig), sien (ban, ban, ban, bun, iri, itu, itu, lig, lig, lig, lig, lig, lig, lig, lig, l-o, l-o, mit, o-z, o-z, o-z, o-z, o-z, o-z, o-z, o-z, o-z, irur, irur, irur, irur, irur, irur), sienac (irur), sienak (l-o), sienian (l-o, o-z), ziên (sur), zieñak (sur), ziela (a-s, bes, gam), zielaïc (lan), zielaïk (garr), zielaic (bes), zielaik (urk), zielaküan (d-b), zielakotz (bar), zielaric (dom), zielarik (buz, gam), zien (aiz, alo, ürr, ürr, ürr, ürr, ürr, a-s, a-s, a-s, azka, bar, bar, bar, bas, bas, bas, bas, bes, bes, bes, d-s, buz, buz, buz, buz, buz, buz, buz, buz, buz, d-b, d-b, d-b, d-b, ezt, gam, gam, gar, gar, gar, garr, iho, izp, jats, lak, lekb, lekb, lekb, lan, lan, larz, larz, larz, larz, larz, larz, larz, leko, mit, mit, sur, sur, sur, dono, dono, dono, bai, dom, suh, u-g, u-g, u-h, urk, urk, urk, urk, urk, mil), zienac (arh, ürr, azka, bas, bes, lan, larz, larz, larz, dono, dom, u-h), zienak (bar, d-b, d-b, d-b, gam, gam, gar, garr, garr, mit, sur, urk, mil), zienen (gar), zienian (alo, alo, d-b, iho, sur, dono, bai), zienin (bar, bes, urk), ciénian (aint)

zienan (1): siénan (l-s)

zieten (1): cieten (mug)

ziezien (8): ciécién (iba), ciecien (hoz, jut, mend, arro), ziezien (d-s, izp, jats)

ziezin (1): ziezin (gam)

ziezte (36): çiezte (luk), ciesté (beh), cieste (am-s, amo, eih, esp, lar, d-g, ure), ciezté (izt, don), ciezte (anh, bard, g-zo, hoz, iri, iru, jut, leko, mug, ora, dona, suh), siésté (kan), zieste (aiz, a-s, beho, dono), ziezte (d-s, buz, iho, jats, lak, lan, larz, u-g)

ziezu (1): béharciéçu (ath)

ziezun (48): errantziezun (izp), çieçün (g-z, g-z), ciéçoun (iba), ciéçun (alt, g-z), cieçoun (jut), cieçun (ainh, ainh, altz, altz, etx, ida, laka, laka, laka, larr, larr, l-z, l-z, mau, mau, mau, men, men, arro, ath, ath, bil, bil), ciecun (etx), ciessoune (ost, ost), ciessun (mend), ciezun (hoz), zeïzun (garr), zeizün (d-b), zeizun (garr), ziezun (alo, alo, bar, bar, d-s, esk, esk, jats, lak, lak)

ziin (21): ciine (ost, ost), ziin (aih, bas, hel, hel, hel, hel, hel, hel, hel), zi-in (aih, aih, aih, aih, aih), ziinac (hel, hel, hel), zi-inac (aih), ziinian (hel)

ziizte (5): siiste (ald), ziizte (azka, bid, hel), zi-izte (aih)

zin (37): ikhoussicin (pag), zaldiezin (garr), cin (arb, arh, arü, arü, arü, sarr, sarr, ünd, gab, gab, gab, las, las, l-o, mit, mug, pag), sin (arh, ürr, lig, mit, urd), zîn (bai, bai), zin (ürr, bid, sarr, ünd, ünd, ezt, gar, garr, mit, dom, dom)

zioat (2): cioat (hir), zioat (uzt)

zioen (5): cioéla (a-z), cioelaric (azk), zioela (hal, jat), zioen (a-s)

zioiat (2): cioyat (get), zioyat (sar)

zion (7): cion (azk, izt, u-g, uzt), ciona (lah), sion (urd), ziola (sur) 
zioozun (1): zioozun (d-s)

zioteiten (1): cioteiten (bida)

zioten (69): emancioten (hir), erranzioten (sur), etzioten (hal, iru, jat), çioten (luk, ur-b), ciotén (ain), cioténn (hen), cioten (ahe, ame, arb, arr, azk, azk, bida, bida, bir, eih, esp, esp, esp, haz, haz, its, its, lah, lah, lar, luh, meh, meh, meh, d-g, d-i, d-i, sen, sen, hir, hir, u-g, urr, urr, uzt, uzt), zioten (ahe, ahe, arb, arr, a-s, bas, bas, zib, zib, zib, hal, hal, jat, jat, luk, d-l, d-l, d-l, sar, sar, sur, dono, mil)

ziotzaten (1): ziotzaten (zib)

ziozkaten (9): ciozcatén (ain), ciozcaten (get), ciozkaten (arb, arr, sen), ziozcaten (bas), ziozkaten (ahe, d-l, sar)

ziozten (1): ciozten (bir)

ziozun (1): cioçun (etxa)

zirade (1): cirade (z-d)

ziradie (3): ciradie (g-i, ida, arro)

ziradien (1): ciradien (müs)

zire (1): siré (itu)

ziren (714): ciélaïc (aint), bacien (leko), Bacien (anh, a-z, arü, bir, lar, osp, l-a, urd, z-d, ur-b), bacirén (ain), baciren (lek, haz, urr), Baciren (zar, arb, arr, azk, b-l, bida, iru, izt, its, l-b, las, mak, hir, dona, ure, uzt), basien (l-o), Basiren (kan), Bazi 'en (ban), bazien (dom, u-h), Bazien (aiz, sur), bazi-in (aih), baziren (ahe, bas, zib, hal, jat, d-l, sar), Baziren (ame, bid, buz, lan, meh, mug, bai), érorissien (bun), erorizien (sur), éroriçienn (hen), eroriciren (ort), eroriziren (aiz), essestatuziren (aiz), etciren (soh), galdouçirènn (bit), haizielaik (sur), haïciren (mug), haiciren (esp), haricirelaric (hir), hari_cirelaric (ald), helduciénac (itu), heldu_cirelaric (mar), helduzienak (sur), jintencien (ünd), jitenciren (mit), norcien (iba), norciren (get), nortzien (sur, gam, iho, larz), nortciren (izt), nurtcien (gab), parthitoussiren (bun), çirélaïc (bit), çirènn (bit, bit), çirelaco (izt), çiren (luk, luk), çirenac (ald), cirén (aiz, ain, ain, ain, ain, ain, ain, don), cirénac (ain, aint, zar, ainh, ainh, ainh, bit, hen, mug), cirénak (don), cirénan (hen), cirénec (hen), cirènn (bit, bit, hen), cirénn (hen, hen), cire (hen), cirelaik (d-g), cirelacoan (hir), cirelaik (bil), cirelakotz (arb, arr, meh), cirelarik (arb, arr), ciren (a-m, aint, arn, eih, zar, zar, zar, ahe, ahe, ahe, ainh, ame, am-s, am-s, am-s, am-s, am-s, am-s, am-s, amo, amo, amo, amo, amo, amo, amo, anh, arb, arb, arb, arb, arb, arb, arr, arr, arr, arr, arr, arh, arh, arh, arm, arü, arü, arü, arü, azk, azk, azk, azk, azk, azka, azka, azka, b-l, b-l, b-l, b-l, b-l, b-l, be-l, be-l, bid, bid, bid, bida, bida, bida, bida, bida, bida, bida, bida, lek, lek, lek, lek, lek, lek, lek, soh, soh, sarr, sarr, sarr, sarr, ünd, ünd, ünd, ünd, ünd, esp, esp, esp, etxa, etxa, gab, get, get, haz, haz, haz, haz, haz, haz, haz, hoz, hoz, ida, ida, iri, iri, iri, iri, iru, iru, iru, iru, iru, iru, izt, izt, its, its, its, its, its, laka, laka, lah, lah, lah, lah, lah, lah, larr, lar, lar, lar, lar, las, las, las, las, las, ald, luk, mak, mak, mak, mak, mak, mak, mak, mar, mar, mau, meh, meh, meh, meh, meh, meh, mug, mug, mug, mug, mug, mug, müs, ora, ora, ora, ora, ora, urd, urd, urd, urd, urd, urd, ort, ort, ort, ort, arro, arro, s-g, s-g, s-g, s-g, s-g, s-g, s-g, d-g, d-g, d-g, d-g, d-g, d-i, d-i, d-i, d-i, d-i, sen, sen, sen, sen, sen, sen, don, don, don, hir, hir, hir, hir, z-d, z-d, z-d, z-d, dona, dona, dona, dona, dona, dona, suh, suh, suh, suh, suh, u-h, u-h, u-h, u-h, ure, ure, ure, ure, ure, ure, urr, urr, urr, urr, urr, urr, urr, uzt, uzt, uzt, uzt, uzt, mil, bil, bil, z-p), cirena (bil), cirenac (am-s, amo, anh, anh, arb, arn, arr, b-l, b-l, b-l, bid, eih, lek, lek, 
esk, etxa, etxa, get, get, haz, hoz, iru, izt, its, its, its, mak, mak, mak, meh, mug, ora, ort, ort, s-g, d-g, d-g, d-i, d-i, hir, dona, suh, suh, suh, ure, ure, ure, uzt, uzt, bil, z-p), c-irenac (mug), cirenak (ahe, arb, arr, azk, bida, bida, esp, men), cirenean (arb, arr, get, iri, its, mug, uzt, uzt), cirenec (las, urr), cirener (arro), cirenian (hal, ida, jat, larr, ora, s-g, z-d, suh, suh, urr), cirenin (urd), cirienian (osp), izanzirenian (a-s), sirén (bun, itu, itu, itu, itu), sirénac (kan, kan), sirénian (kan, itu), sirelarik (luh), siren (ürr, ürr, ürr, ban, ban, lek, kan, kan, kan, kan, kan, kan, kan, kan, itu, itu, ald, ald, ald, l-o, l-o, l-o, l-o, luh, luh, luh, mit, mit, mit, mit), siren- (kan), siren) (itu), sirenac (luh, luh), sirenian (ürr, mit), tziren (bas), zirelaco (d-s), zirelacotz (iri), zirelaic (ezt), zirelakotz (ezt, sar), zirelaric (lak, u-g), ziren (ahe, ahe, ahe, ahe, ahe, alo, alo, ame, ame, ame, ame, ame, arr, arh, beho, ürr, ürr, a-s, a-s, a-s, a-s, a-s, a-s, bar, bid, d-s, buz, zib, zib, zib, zib, zib, zib, zib, zib, esk, esk, esk, esk, ezt, ezt, ezt, ezt, ezt, ezt, gar, gar, gar, gar, gar, hal, hal, hal, hal, hal, hal, iho, iho, izp, izp, izp, jat, jat, jat, jat, jat, jat, jats, jats, jats, lak, lak, lekb, lekb, lekb, lekb, lekb, lekb, lekb, lekb, lan, luk, luk, luk, d-l, d-l, d-l, sar, sar, sar, sar, sar, sar, sar, dono, dono, bai, bai, bai, bai, bai, bai, bai, dom, dom, dom, dom, dom, u-g, u-g, u-g, u-g, u-g, u-h, mil, mil, mil), zirenac (aiz, ame, ame, esk, ezt, ezt, hal, hal, hal, jat, jat, jat, lak, lak, lak, lekb, lekb, lan, luk, luk, luk, bai, bai, dom, u-g, u-g), zirenak (ahe, alo, alo, a-s, a-s, a-s, bar, d-s, d-s, buz, buz, ezt, gar, gar, jats, jats, lekb, sar, mil, mil), zirenean (arb, arr, mil), zirenec (iho), zirenek (sar), zireneri (zib, d-l), zirenian (aiz, d-s, lekb, luk, bai), zirenin (esk)

zirete (3): cirete (its), zirete (hal, jat)

zirezte (4): cirezte (lek, arn), zirezte (izp, mil)

zirezun (2): sireçun (lig), zirezun (esk)

zirie (5): cirié (ainh), cirie (be-l, soh, osp, osp)

zitain (1): zitain (urk)

zite (4): cité (a-zo, altz), cite (g-zo, laka)

zitekeen (1): citekeen (b-l)

ziten (17): sarciten (lar), sartciten (hoz), sartziten (iho), citénn (hen), citelaric (its), citen (beh, bid, iru, ora), siten (lah), ziten (bas, buz, izp, lak, bai, mil), zitiela (garr)

zitezkeien (1): zitezkeyen (ahe)

zitian (1): etcitian (ure)

zitien (13): citien (a-m, am-s, amo, leko, ora), tsén (l-s), tsitien (ban), zitién (bun), zitien (aih, garr, garr), zitienak (gam), zitienian (garr)

zitiin (2): etzitien (buz), ziti-in (aih)

zitin (7): etzitiin (hel), etcitin (arr), etzitin (bid), etcitin (las), citin (altz), zitîn (bai), zitin (ezt) zitititzun (1): ezitititzun (bar)

zititzien (8): écititcien (alt), etzititzien (alo), etcititcien (larr, bil), ezcitcien (iba), cititcien (men, bil, bil)

zititzun (3): etcititçun (ida), etçititçun (laka), etcititçun (l-z)

zitizien (40): etciticien (men), étciticién (ost), etciticien (arro, ath, altz, ainh, etx, l-z), citiciën (g-z, g-z), citicien (ainh, ainh, alt, alt, altz, altz, etx, etx, ida, laka, laka, larr, l-z, l-z, mau, mau, men, arro, s-g, ath, ath), cititcien (ida, larr), zititzien (iho), zitizien (alo, alo, bar, bar, esk, esk)

zitizun (4): etsitiçün (g-z), etciticun (etx), etcitiçun (mau), etzitizun (esk)

zituen (29): étsitouen (kan), étcitouén (ain), etzituen (bir), etcituen (izt), etzituen (jat), citouén 
(ain), citouen (sen, dona), citouenn (hen), cituen (ame, azk, b-l, bida, bida, bida, esp, get, its, mak, hir, urr), cituena (lar), sitouen (ald), zituen (aiz, ahe, zib, d-l, sar), zituena $(\mathrm{d}-\mathrm{l})$

zituin (1): etzituin (hal)

zituzten (104) : etciousten (aha), etciouzten (aint), etcitusten (arb), etzituzten (larz), ezcituzten (get), ézituzten (ur-b), çitusten (ur-b), citousténn (hen), citousten (arh, arh, ald, eih), citouztén (ain, ain, ain), citouzten (lah, lah, sen, sen, dona, dona), citusten (arn, arb, azk, azk, esp, esp, lar, mak, u-h, u-h), cituzten (arn, arr, bida, lek, lek, get, get, get, haz, haz, haz, haz, iri, iru, izt, izt, its, its, las, las, las, mak, meh, meh, meh, mug, mug, hir, hir, hir, urr, urr, urr, uzt, uzt, uzt), cituztenetarik (lek), sitoustén (bun), sitousten (kan, kan, ald, luh), situsten (ur-b, ur-b), zitouzten (buz, buz, buz, gam), zitusten (ame, beho, zib, jat), zituzten (ahe, ahe, ame, arb, arr, aih, bas, zib, ezt, hal, hal, jat, larz, meh, d-l, d-l, sar, sar, bai)

zituzun (1): citouçoun (arm)

zitzaioten (1): citçayoten (hir)

zitzaizkoten (1): zitzaizkotela (zib)

zitzien (45): etcitcien (beh), etzitzien (bes), etcitzien (hoz), etzitzien (iho), Etzitzien (bes), Etcitcien (etxa), eztcitcien (etxa), citcién (iba, ost), citcien (a-zo, beh, beh, bard, g-zo, etxa, etxa, gab, gab, hoz, iba, jut, jut, ost), citsien (mend, mend), citzien (hoz), sitcien (bard), tcitcien (gab), zitzien (bes, bes, d-s, d-s, d-b, d-b, gam, gam, garr, garr, iho, izp, izp, jats, jats, lak, lak)

zitzin (9): etcitcin (g-zo), etzitzin (izp, jats), citcin (arm, arm, arm, jut), zitzin (d-s, lak)

zitzun (71): Baçitçoun (arm), Bacitçun (gab), Bacitzoun (iba), bacitzun (iho), Bacitzun (hoz), Bazitzün (d-b), bazitzun (d-s, izp), Bazitzun (jats, lak), aicitçun (mar), çitçun (gab), çitçun- (g-zo), citçoun (a-zo, arm, arm, arm, iba, iba, jut, jut, jut), citçounn (jut), citçun (a-zo, a-zo, a-zo, beh, beh, beh, beh, g-zo, g-zo, g-zo, g-zo, gab, gab, gab, mar, mar), citçun) (a-zo), citsoune (ost, ost), citsun (mend, mend), citzun (hoz, hoz, hoz), zitzoun (gam), zitzun (d-s, d-s, d-s, d-b, d-b, garr, garr, iho, iho, iho, iho, izp, izp, izp, izp, jats, jats, jats, lak, lak, lak, lak, lak)

ziuten (1): etziouten (a-s)

ziutzun (7): baziutzun (bes), ziutzun (bes, bes, bes, bes, urk, urk)

ziuzte (1): eziuzte (urk)

ziuzten (86): etziusten (dono, azka), etciuzten (ort), etziuzten (sur, dom), etciuzten (u-g), etziuzten (l-o), ezciuzten (mug), eziuzten (lan), ciouzten (zar, zar, jut), ciouztenac (iba), ciusten (am-s, amo, azka, b-l, b-l, bir, lek, iri, mak, d-i, d-i, d-i, ure, ure), ciuzten (anh, anh, anh, anh, anh, bid, bid, iru, lah, leko, leko, mug, ort, ort, suh, suh), siusten (ban, ban, ban), siustenac (mend), ziousten (a-s, azka, hel), ziouzten (a-s), ziusten (sur, dono, dono, mil, mil), ziuzten (azka, bas, bas, bas, bes, ezt, lak, lan, larz, ort, sur, sur, bai, dom, suh, urk, mil), ziuztenac (ezt, lan, suh), ziuztenak (jats), ciousten (aha, eih), cioustenac (aha), ciouzten (aint), ciouztenac (aint), ciuzten (a-m),

ziuzteten (1): ziousteten (hel)

zizen (1): ciçen (men)

zizien (138): étsissien (bun), etsicien (g-z), Etsicien (bard), Etcisiën (g-z), Etcissién (ost), etcicien (mend, bard, mar), etzizien (bar), etzicien (iho), etzizien (izp), etcicien (altz), etzizien (bes), etcicien (mau, men), etcicién (ost), etcicien (arro), etzizien (urk), etcicien 
(a-zo), étcicien (z-p), etcicien (bard, g-zo), etzizien (d-b, esk), etcicien (ainh), etzizien (gam, garr), etcicien (hoz), étcicien (iba), etcicien (ida), etzizien (iho, alo, izp, jats), etcicien (laka, larr, bil), Etcicien (mau, men, mend, arro, d-g, ath, a-zo, z-p), etcicien (altz), Etcicien (beh), Etzizien (bar), Etcicien (g-zo), Etzizien (d-b, esk), Etcicien (ainh, etx), Etzizien (gam), etzizien (garr), Etcicien (alt, hoz), Etcicién (iba), Etcicien (ida), Etzicien (iho), Etzizien (alo, izp, jats), Etcicien (laka, larr, l-z, mar, bil), eztcicien (etxa), Eztcicien (gab), cicién (ainh, ost, ost), cicien (ainh, alt, alt, a-zo, altz, altz, beh, beh, beh, bard, g-zo, g-zo, etx, etxa, gab, gab, hoz, hoz, iba, iba, jut, jut, jut, jut, laka, larr, osp, l-z, mar, mar, mau, mend, mend, mend, arro, arro, d-g, ath, bil), tcicien (gab), zizien (alo, bar, bes, d-s, d-s, d-s, d-s, d-b, d-b, esk, esk, gam, garr, iho, iho, izp, izp, jats, jats, lak, lak, lak), Etcicien (a-m)

zizin (34): etcicin (a-zo), çiçinn (bit), cicin (a-zo, arm, arm, arm, arm, arm, arm, arm, bard, bard, iba, iba, jut, jut, jut, men, mend, mend, mend), cicine (ost), cicinn (beh), zizin (d-s, d-b, d-b, gam, gam, garr, iho, izp, jats, jats, lak)

zizte (34): cisté (a-zo, l-s, ost), ciste (aha, arh, mar, mend, d-i, u-h), cizté (aint, zar, iba, l-b), cizte (a-m, a-z, arm, gab, ilh, las, izu), sisté (bit, bun), siste (ban, ban, luh), zîzte (bai), zizte (bes, d-b, ezt, gam, garr, lekb, sur, dom, urk)

zizten (1): zizten (d-b)

zizun (49): éraïtencisun (etx), etzizun (gam), hartuzizun (alo), çiçun (ainh, alt, alt, etxa, laka), ciçün (g-z), ciçun (ainh, altz, altz, etx, ida, lig, larr, larr, l-z, l-z, mau, mau, mau, men, arro, arro, arro, s-g, s-g, s-g, ath, ath, ath, bil, bil, bil), cicün (g-z), ziçun (men), zizun (alo, alo, alo, bar, bes, esk, esk, garr, iho, izp, lak, urk)

zoan (9): çoala (a-z, azk, ure), çoan (ort), zoala (beho, bir, izp, jats, luk)

zohan (19): çohala (ain, bida, esp, its, mak, hir, urr), sohala (ban, hoz), zohala (ahe, arb, arr, bas, get, hal, jat, sar, uzt), zouhala (buz)

zoon (1): alhantzoon (larz)

zu (39): beldourtzou (gam), beldourtçou (iba), béldurtçu (ost), beldurtzu (d-s, iho), beldurçu (mend), beldurzu (garr), etzou (gam), étçu (beh), etzu (izp, hoz), gaichtouactçu (a-zo), gachtotçu (ost), gachtouaktzou (gam), çou (bit), çu (a-zo, beh, beh, g-zo), sou (bun), tçou (iba, jut, jut, jut), tçu (a-zo, a-zo, beh, beh, beh, beh, beh, g-zo, g-zo, g-zo, mar), tzu (d-b, izp), zu (beh, garr)

zuan (8): baçuala (gab), çüala (las), zuala (azka, iho, larz, d-l, bai, ur-b)

zuen (121): çouéla (aint), baçouela (ainh), éguitencuen (ur-b), erraitentzuen (aiz), etzuen (luk), çouén (ain), çouënn (hen), çouénn (hen), çouela (a-m, zar, sen), çouelaric (sen), çouen (ain, ain, lah, lah, sen, sen), çouenn (hen), çuelaric (mug), çuen (azk, azk, b-l, b-l, bida, bir, lek, lek, lek, get, iru, iru, iru, its, its, its, lar, mak, mak, mak, mak, meh, ort, ort, d-i, hir, hir, ure, ur-b, ur-b, urr, urr, urr), çuenak (bida), çuhela (iru), cuen (di, d-i), souéla (l-s), souen (kan, kan, kan, ald, ald, luh, luh), suen (ur-b), zouen (arh), zuela (d-s, lak), zuelaric (lan), zuelarik (haz), zuen (ahe, ahe, ahe, ame, ame, ame, arb, arb, arr, bas, bir, bir, bir, lek, zib, zib, get, get, hal, hal, hal, haz, haz, haz, haz, iri, iri, iri, jat, jat, jat, lan, luk, meh, meh, d-l, d-l, d-l, d-l, sar, sar, ur-b, uzt, uzt, mil, mil, mil, mil, mil), Zuen (sar)

zuin (2): haizuin (sur), ikusizuin (sur)

zun (2): çun (beh), zun (garr)

zuten (463): causituçuten (hir), çoutélacotz (hen), çoutelaïc (aha, arm), çoutélaric (ain, ain, 
hen), çoutelaric (sen, dona), çouten (aha, aint, eih, zar, zar, arh, lah, lah, lah, ora, ora, sen, sen, dona, dona, dona, dona, dona), çoutén (ain, hen, don), çoutenean (sen), çoutenian (aha, arm, iba, jut), çouténian (don), çouteniann (bit), çoutènn (bit, bit, hen), çoutenn (hen), çouténn (hen), çutela (ure), çutelacotz (lar), çutelaic (d-i), çutelakots (esp), çutelaric (azk, b-l, b-l, hoz, iru, las, ort, d-g, urr), çutelarik (esp), çuten (a-m, eih, am-s, am-s, am-s, am-s, amo, amo, amo, amo, anh, anh, anh, a-z, a-z, a-z, arh, azk, azk, azk, azk, b-l, b-l, b-l, bida, lek, lek, lek, lek, lek, esp, esp, esp, esp, esp, esp, haz, haz, haz, haz, haz, ilh, iru, iru, iru, iru, izt, izt, izt, its, its, its, its, its, l-b, l-b, lah, las, las, mak, mak, mak, mar, meh, leko, leko, leko, leko, mug, mug, ort, ort, ort, ort, izu, izu, d-g, d-g, d-i, d-i, d-i, don, hir, hir, hir, hir, u-g, u-g, u-g, ure, ure, ure, urr, urr, urr, urr), cuten (anh, azk, lek, gab, lar, mar), çutén (don, don), çuténa (a-z), çutena (a-zo, g-zo, müs), çutenean (bida, iru, leko, ort, hir), çutenetaric (b-l), çutenian (a-m, azk, hoz, ilh, l-b, luk, mak, mend, izu), çuténian (a-z, beh), cutenian (mar), çutenn (hen), etçouten (aha, aint, ora, zar), Etçoutén (ain), étçoutén (ain), Etçouten (ora, sen, u-g), etçoutènn (bit), étçoutènn (bit), Etçuten (aint, eih, a-z, ilh, las, l-b, müs, izu, ure, zar), etçuten (a-m, bida, leko, müs, ilh, izu, d-g, a-z, lar, las, l-b, mar, ure), Etçutènn (bit), Etsoutén (bun), étsouten (bun, kan), Etsutén (itu), Etsuten (kan, l-s), étsutén (l-s, l-s), etuten (dono), Etzouten (aha, a-s, buz), etzouten (arh, a-s, buz, hel), etzuten (aiz, aiz, arr, azka, bid, bir, hal, iru, jat, lan, larz, l-o, luk, luk, sur, bai, dom, arb, u-g), Etzuten (aiz, arr, azka, bid, hel, hal, iru, jat, lan, larz, l-o, sur, dono, bai, dom, arb), etzuzten (a-m), ezçuten (d-i), ezcuten (mug), ezt'suten (ban), eztçuten (izt), izantzutenian (sur), izatuzuten (ur-b), oukhançoutènn (bit), oukhanzouten (a-s), soutelaric (ald), soutén (bun), soutèn (bun), souten (kan, kan, hel, ald, ald, ald, ald, luh), souténiane (ost), souténiann (hen), sutelaïc (ban), sutelarik (l-o), suten (ban, itu), sutén (itu, l-s), suténia (ur-b), t’zuten (ban), tzuten (uzt), Yoçuten (ilh), yozouten (as), Yozuten (aiz), yozuten (sur, azka), zoutelaïc (hel), zouten (arh, arh, buz, hel, lekb, lekb, lekb), zoutenian (buz, gam, hel), zutela (ahe, izp), zutelacotz (zib), zutelaic (bai), zutelaik (azka), zutelaik (garr), zutelakotz (sar), zutelaric (arn, beho, ezt, sar, u-g), zutelarikan (mil), zuten (aiz, ahe, ahe, ahe, ame, ame, ame, ame, arb, arb, arb, arr, arr, arr, azka, azka, aih, aih, aih, aih, aih, bas, bas, bas, bas, bid, bid, bid, bir, bir, bir, buz, zib, zib, zib, ezt, ezt, ezt, ezt, ezt, get, get, get, get, hel, hal, hal, hal, iri, iri, iri, iri, jat, jat, jat, lan, larz, larz, l-o, luk, luk, luk, luk, meh, meh, meh, mug, d-l, d-l, d-l, d-l, sar, sar, sar, sar, dono, dono, dono, bai, bai, dom, dom, dom, suh, suh, suh, u-h, u-h, u-h, u-h, u-h, urk, urk, ur-b, uzt, uzt, uzt, mil, mil, mil, arn, beho), züten (d-b), zutena (beho, d-s), zutenean (ahe, get, lak, d-l, sar), zutenetic (hoz), zutenian (aiz, aih, bas, bid, d-b, garr, izp, lan, larz, meh, dono, beho)

zutian (11): etsutian (irur, z-p, lig), etçutian (müs, s-g, g-i), etzutian (l-a), ezçutian (osp), çutialaric (be-l, soh), çutian (l-a)

zutien (67): etsutien (o-z), etzutien (mit), etçutien (z-d), etzutien (ürr), etçutien (soh), etzutien (gar), étçutien (hau), çütienac (g-z), çutién (hau), çutien (arü, be-l, be-l, soh, soh, g-i, g-i, hau, osp, osp, l-a, l-a, müs, müs, urd, urd, pag, pag, s-g, z-d, z-p, z-p), çutienac (soh, laka, osp, mau, z-d), çutienak (ath), cutien (z-d), cutienac (be-l, pag), sutien (lig, lig, lig, mit, o-z, o-z, irur, irur, irur, irur), sutienac (ürr, z-p), sutinac (mit), tcutien (bel), zoutien (lekb), zutien (ürr, ürr, sarr, sarr, ünd, ünd, gar, gar, mit), zutienac (esk), zutienak (gar), zutieneko (zib) 
zutin (6): etçutin (pag, arü), etzutin (sarr, ünd), çutila (altz), çutin (arü)

zututzen (1): aiçûtutcen (alt)

zututzun (21): çututçün (g-z), çututçun (ida, ida, ida, men, men, men, men, men, ath, ath, ath, ath, bil, bil, bil), zututzun (alo, alo, esk), Baçututçün ( $g-z)$, Bazututzun (esk)

zutuun (1): çutuün $(g-z)$

zutuzen (2): çutucen (alt), cütutcen (alt)

zutuzun (51): Baçutuçun (altz, laka), bazutuzun (bar), arizutuzun (esk), çutuçün (g-z, g-z, g-z, g-z), çutuçun (ainh, ainh, ainh, altz, altz, altz, etx, etx, etx, etxa, etxa, etxa, etxa, ida, laka, laka, laka, laka, laka, larr, larr, larr, larr, larr, l-z, l-z, l-z, mau, mau, mau, mau, arro, arro, arro, arro, arro, ath), zutuzun (alo, bar, esk, esk, esk, esk)

zutzun (3): çutçun (bard, bard, bard)

zuun (2): çououne (ost), sououn (ost)

zuzien (2): zuzien (urk, urk)

zuzten (65): étsusten (itu), etçuzten (izu, a-z, zar), etzüzten (d-b), etçuzten (ilh), etcuzten

(l-b), ezuzten (lekb), çousténac (bit), çoustènn (bit), çousten (ora), çouztén (don), çouzten (zar, arm, ora), çusténak (beh), çusten (am-s, amo, eih, etxa, ilh, l-b, izu, d-g, d-g), çuzten (a-z, a-z, ilh, mar, izu, izu), çuztenac (luk), çuztenak (ilh, l-b), custen (lb), cuztenak (bard), soustén (ost), sousténac (bun), sustélaïc (itu), sustén (itu, itu, l-s, l-s), suzten (l-o, l-o, l-o), züztenak (d-b), zousten (arh, lekb), zouzten (a-s, u-g), zusten (ame, beho, bir, luk, u-h), zuzten (aih, lekb, larz, bai, u-g, urk, urk), zuztenak (mil)

zuzu (2): baçuçu (ath), çuçu (men)

zuzun (379): aïçuçun (alt), baçun (mar), etzuzun (bar), -houraçouçoun (arm), ohartuzuzun (bes), choutitouçouçoun (arm), çouçoun (arm, arm, arm, arm, arm, arm, bit, bit, bit, iba, iba, iba, iba, iba, iba, iba, iba, iba, jut, jut, jut, jut, jut, jut, jut, jut, jut), çuçün (g-z, g-z, g-z, g-z, g-z), çuçun (ainh, ainh, ainh, ainh, ainh, ainh, ainh, ainh, alt, alt, alt, alt, alt, a-zo, a-zo, a-zo, a-zo, a-zo, a-zo, a-zo, altz, altz, altz, altz, altz, altz, beh, beh, beh, beh, beh, beh, beh, beh, beh, beh, bard, bard, bard, bard, bard, bard, bard, bard, g-z, g-zo, g-zo, g-zo, g-zo, g-zo, g-zo, etx, etx, etx, etx, etx, etx, etx, etxa, etxa, etxa, etxa, etxa, etxa, etxa, etxa, etxa, gab, gab, gab, gab, gab, gab, ida, ida, ida, ida, ida, ida, ida, ida, laka, laka, laka, laka, laka, laka, laka, laka, laka, laka, lig, larr, larr, larr, larr, larr, larr, larr, larr, larr, l-z, l-z, l-z, l-z, l-z, l-z, l-z, l-z, l-z, l-a, mar, mar, mar, mau, mau, mau, mau, mau, mau, mau, men, men, men, men, men, men, men, men, mend, arro, arro, arro, arro, arro, arro, arro, arro, arro, s-g, s-g, ath, ath, ath, ath, ath, ath, ath, bil, bil, bil, bil, bil, bil, bil, bil, bil), çuçun- (mau), çucün (g-z), çucun (etx, etx), çuzun (hoz, hoz, hoz, hoz, hoz, hoz, hoz, hoz, hoz), cuçün (g-z), cuçun (gab), izantzuzun (iho), souçoune (ost), soucoune (ost), soussoune (ost, ost, ost, ost, ost), suçun (mend), susun (mend, mend, mend, mend, mend), tçuçun (gab, gab), yarriçouçoun (arm), züzün (d-b, d-b, d-b, d-b), zouzoun (gam, gam, gam, gam, gam, gam, gam), zuzun (alo, alo, alo, alo, alo, alo, alo, bar, bar, bar, bar, bar, bar, bar, bar, bes, bes, bes, bes, bes, bes, bes, bes, d-s, d-s, d-s, d-s, d-s, d-s, d-s, d-s, d-s, d-s, d-b, d-b, d-b, d-b, d-b, esk, esk, esk, esk, esk, esk, esk, garr, garr, garr, iho, iho, iho, iho, iho, iho, iho, iho, iho, iho, izp, izp, izp, izp, izp, izp, izp, izp, izp, jats, jats, jats, jats, jats, jats, jats, jats, jats, lak, lak, lak, lak, lak, lak, lak, lak, lak, urk, urk, urk), baçouçoun (arm, iba, jut), Baçouçoun (jut), baçuçün (g-z), baçuçun (ainh, a-zo, bard, g-zo, etx, etxa, gab, ida, laka, l-z, mau, men, mend, arro, d-g, u-g, bil), Baçuçun (ainh, a-zo, beh, bard, g-zo, etxa, ida, larr, l-z, 
mar, men, mend, arro, ath, bil), Baçucun (alt, etx), baçuzun (hoz), Bassoussoun (ost), bassoussoune (ost), bazouzoun (gam), Bazouzoun (gam), bazuzun (alo, bes, d-b, esk, garr, izp, jats, lak), Bazuzun (alo) 
